\title{
Mode coupling in spin torque oscillators
}

\author{
Steven S.-L. Zhang ${ }^{\mathrm{a}}$, Yan Zhou ${ }^{\mathrm{b}, \mathrm{c}}$, Dong Li ${ }^{\mathrm{d}}$, Olle Heinonen ${ }^{\mathrm{e}, \mathrm{f}, \mathrm{g}, *}$ \\ ${ }^{a}$ Department of Physics and Astronomy, University of Missouri, Columbia, Missouri \\ 65211, USA \\ ${ }^{b}$ Department of Physics, The University of Hong Kong, Hong Kong, China \\ ${ }^{c}$ Center of Theoretical and Computational Physics, Univ. of Hong Kong, Hong Kong, \\ China \\ ${ }^{d}$ Department of Physics, Centre for Nonlinear Studies, and Beijing-Hong \\ Kong-Singapore Joint Centre for Nonlinear and Complex Systems (Hong Kong), Hong \\ Kong Baptist University, Kowloon Tong, Hong Kong, China \\ e Material Science Division, Argonne National Laboratory, Lemont, Illinois 60439, USA \\ ${ }^{f}$ Northwestern-Argonne Institute of Science and Technology, 2145 Sheridan Road, \\ Evanston, Illinois 60208, USA \\ ${ }^{g}$ Computation Institute, The Unversity of Chicago, 5735 S Ellis Ave, Chicago, IL 60637 \\ $U S A$
}

\begin{abstract}
A number of recent experimental works have shown that the dynamics of a single spin torque oscillator can exhibit complex behavior that stems from interactions between two or more modes of the oscillator. Examples are observed mode-hopping or mode coexistence. There has been some intial work indicating how the theory for a single-mode (macro-spin) spin torque oscillator should be generalized to include several modes and the interactions between them. In the present work, we rigorously derive such a theory starting with the Landau-Lifshitz-Gilbert equation for magnetization dynamics by expanding up to and including third-order terms in deviation from equilibrium. Our results show how a linear mode coupling, which is necessary for observed mode-hopping to occur, arises through coupling to a magnon bath. The acquired temperature dependence of this coupling implies that the manifold of orbits and fixed points may shift with temperature.
\end{abstract}

Keywords: spin-torque oscillator

\footnotetext{
${ }^{*}$ Corresponding author

Email addresses: ZhangShule@missouri.edu (Steven S.-L. Zhang), yanzhou@hku.hk (Yan Zhou), geodesic.ld@gmail.com (Dong Li), heinonen@anl.gov (Olle Heinonen)
} 
PACS: 85.75.-d, 76.50.+g, 72.25.-b

\section{Introduction}

Since the prediction of spin transfer torque (STT) in 1996[1, 2, 3], whereby a spin-polarized de current exerts a torque on the local magnetization order parameter, there has been a wealth of theoretical and experimental investigations of phenomena driven by STT. One particular manifestation of STT is the spin torque oscillator (STO). The STO is typically realized in $\mathrm{MgO}$ magnetic tunnel junctions $[4,5,6,7,8]$, or metallic nanocontacts $[9,10,11]$; in both of these, a dc current is driven perpendicularly to two thin stacked magnetic layers, in one of which the magnetization is relatively free to rotate, while in the other the magnetization is held fixed. With the relative magnetization directions and current direction arranged appropriately, STT pumps energy into the STO, and by adjusting the current magntitude, this pumping can be made to cancel the intrinsic dissipative processes in the system. This gives rise to almost undamped oscillations with a very small linewidth. As STOs are potentially useful in technological applications, such as frequency generators or modulators, it is both of practical as well as of fundamental interest to understand the physics of the STO auto-oscillations. Slavin and co-workers[12, 13, 14, 15] put forth a comprehensive theory valid for single-mode STOs, that is, STOs for which one mode is relevant and is excited (this is when a macro-spin model is readily applicable). Some striking features of this theory are the effects induced by the inherent nonlinearity of the STOs, for example the behavior of the oscillator linewidth below and above threshold $[13,14,15]$, which is the current at which STT pumping first cancels damping and auto-oscillations are achieved.

Recently, there have been several experiments demonstrating the effects of multi-mode STOs, for example mode co-existence and mode-hopping[16, $17,18,19,20,21,22,23]$. Clearly, the interactions between several oscillator modes cannot be described by the single-mode theory but requires a theory that describes the interactions between collective modes, and how the behavior of the collective modes is modified as a consequence of those interactions. de Aguiar, Azevedo, and Rezende[24] considered a two-mode system with in-plane translational invariance and arrived at coupled equations similar to those of the Lotka-Volterra biological model. However, de Aguiar and co-authors argued that in the driven two-mode system, one mode 
will get extinguished and only one mode survives. This is obviously not consistent with many experimental observations of mode-hopping and modecoexistence. A multi-mode theory was first outlined by Muduli, Heinonen, and Åkerman[22, 8, 25]. In particular, these authors argued that the equations describing two coupled modes could be mapped onto a driven dynamical system used to describe semiconductor ring lasers[26, 27]. It is known that in the presence of thermal noise, those equations exhibit mode-hopping in certain regions of parameter space[27]. A key observation here was that for mode-hopping to be present in a two-mode system, the time derivative of the slowly varying amplitude of one mode must be coupled linearly to the amplitude of the other mode (a so-called "backscattering" term). Also, the authors gave some general argument for why mode-hopping is a minimum when the free layer magnetization is anti-parallel to that of the fixed layer, and then increases as the orientation moves away from anti-parallel[22]. Later, Iacocca, Heinonen, Muduli, and Akerman[28] also showed that the effective multi-mode theory described very well observed line-width broadening near mode crossings[8], and Sharma et al.[29] observed a $1 / f$-type frequency noise spectrum in magnetic tunnel junction STOs, which was attributed to mode hopping. Iacocca et al.[30] also presented experimental results that identified two mode coupling mechanisms: magnon-mediated scattering, and intermode interactions consistent with preliminary results of the present work[31].

With the mounting experimental observations that appear to be consistent with the multi-mode theory of Muduli, Heinonen, and Akerman[22], it is of interest to present a rigorous derivation of the equations for coupled modes from first principles (the micromagnetic Landau-Lifshitz-Gilbert equation), and to analyze the ensuing behavior of the system. That is one purpose of the present work. We will show how the linear backscattering term arises naturally in a system with a small number, e.g., two, of dominant modes but in which there is a bath of many modes. This bath provides effective interactions between the dominant modes when the bath is integrated out and the equations projected onto the subspace of dominant modes. We will also derive and discuss some consequences of this theory. The backscattering terms have a direct temperature dependence as they involve thermal populations of modes. This has a consequence of a temperature dependence of the magnitude of the backscattering terms, as we will explicitly show in an example, which leads to a temperature dependence of the linewidths of the modes[28]. The temperature dependence of the backscattering terms also have a more subtle consequence in that manifold of orbits and fixed point 
will shift as a function of temperature, which will change the location or height of saddle points that are crossed during mode-hopping.

While the algebra may at times seem a bit tedious, we are writing out some of the expressions explicitly to point out symmetries and physical consequences. We will also present some examples to illustrate how mode hopping can arise from mode interactions. Our results will show that there is always some small possibility of mode hopping, consistent with experimental observations[22]. However, the backscattering term that leads to mode hopping grows with the appearance of nutation in the modes of the system, in which the phase between the dynamical components of the magnetization motion is not constant across the magnetic layer. For a system such as the MTJ with an in-plane magnetic field studied in Ref. [22], we will show how the backscattering increases as the external field is rotated away from the direction of the fixed layer, consistent with experimental observations[22]. Finally, we note that the method we use here is based on an expansion in eigenmodes of the linearized conservative equations of motion for the magnetization and will therefore not apply to, e.g., systems that exhibit a localized bullet mode[32].

\section{Methods}

\subsection{Micromagnetic Equations}

Our starting point is a soft ferromagnetic system, for example a thin film. We describe the local magnetization by a director $\hat{m}_{i}$ for discrete sites $i=1,2, \ldots N$, with $\left|\hat{m}_{i}\right|=1$. The LLG equation including damping and spin torque is then

$$
\frac{d \hat{m}_{i}}{d t}=-\gamma \hat{m}_{i} \times \mathbf{H}_{\mathrm{eff}, i}-\frac{\gamma \alpha}{1+\alpha^{2}} \hat{m}_{i} \times\left[\hat{m}_{i} \times \mathbf{H}_{\mathrm{eff}, i}\right]+\gamma a_{J} \hat{m}_{i} \times\left[\hat{m}_{i} \times \hat{M}\right] .
$$

Here, $\gamma$ is the gyromagnetic ratio, $\alpha \ll 1$ the dimensionless damping, $a_{J}$ the effective field due to STT, and $\hat{M}$ the (uniform) magnetization direction of the fixed layer; the effective field $\mathbf{H}_{\mathrm{eff}, i}$ includes exchange, demagnetizing fields, and an external applied field $\mathbf{H}_{\text {ext }}=H_{\text {ext }, x} \hat{x}+H_{\text {ext }, y} \hat{y}+H_{\text {ext }, z} \hat{z}$. We will not here include Oersted fields generated by the currents in the system as they are not important for the present analysis, although it has shown that these fields play an important role in the interactions between certain modes in nano-contact STOs[23]. We are also ignoring the so-called field-like, or perpendicular, spin torque[33] as this can be absorbed into the definition of 


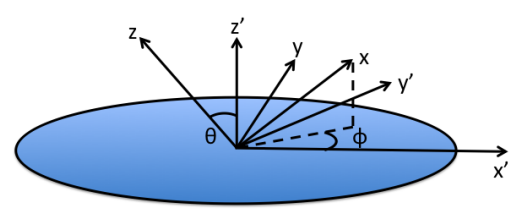

Figure 1: (Color online) Geometry used in this work. The FL is in the $\hat{x}^{\prime} \hat{y}^{\prime}$-plane; the $\hat{x}$-axis is along the FL magnetization equilibrium direction, which may point out of the $\hat{x}^{\prime} \hat{y}^{\prime}$-plane because of the applied field $\mathbf{H}_{\text {ext }}$. The projection of the $\hat{x}$-axis on the $\hat{x}^{\prime} \hat{y}^{\prime}$-plane is rotated an angle $\phi$ from the $\hat{x}^{\prime}$-axis. The $\hat{y}$-axis is in the $\hat{x}^{\prime} \hat{y}^{\prime}$-plane, and the $\hat{z}$-axis is rotated an angle $\theta$ from the $\hat{z}^{\prime}$-axis.

the external field. We shall combine exchange and demagnetizing fields into a single field $\mathbf{H}_{d, i}$ and note that in general we can write

$$
H_{d, i, \delta}=\sum_{i^{\prime}, \epsilon} D_{i, i^{\prime} ; \delta, \epsilon} m_{i^{\prime}, \epsilon}, \delta, \epsilon=x, y, z
$$

where $D_{i, i^{\prime} ; \delta, \epsilon}$ is a generalized demagnetizing tensor that includes near-neighbor exchange. We shall also assume that the magnetoscystalline anisotropy is negligible, and we take the $\hat{x}$-axis to be along the average equilibrium magnetization in the free layer. Without loss of generality, we can take the $\hat{y}$-axis also to be in the $\hat{x}^{\prime} \hat{y}^{\prime}$-plane. Figure 1 illustrates the geometry of the system. In general, the demagnetizing tensor will in the representation of the $x y z$ coordinate system have all off-diagonal terms non-zero. However, for some specific examples such as magnetic tunnel junctions with an in-plane external field, or systems with a large $\left(H_{\text {ext }} \gg 4 \pi M_{S}\right)$ external field perpendicular to the planes of the magnetic layers, the demagnetizing tensor can be taken to be (approximately) diagonal in the $x y z$ coordinate system. In such cases, if the external field is in the $\hat{x}^{\prime} \hat{z}^{\prime}$-plane, the only off-diagonal elements are $D_{i, i, x, z}=D_{i, i^{\prime} ; z, x}$. Figure 2 depicts the equilibrium magnetization in the free layer of a circular magnetic tunnel junction STO of diameter $d=240 \mathrm{~nm}$ obtained from micromagnetic simulations with parameters appropriate for the systems in Ref. [34]. In the figure, the pinned layer and reference layer 


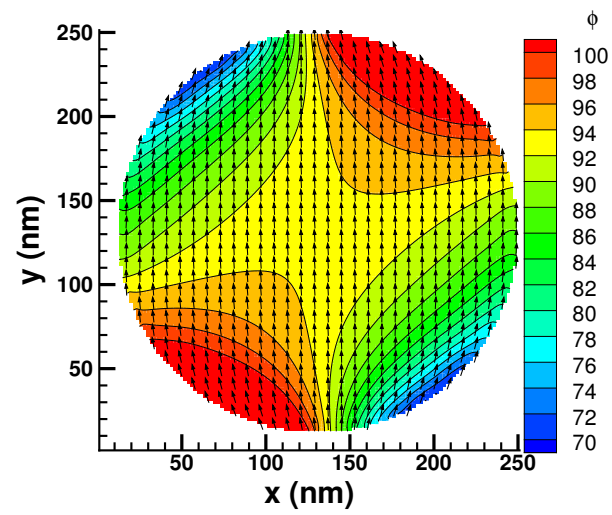

Figure 2: (Color online) Micromagnetic equilibrium magnetization in the free layer of a circular magnetic tunnel junction of diameter $240 \mathrm{~nm}$. The pinned and reference layer magnetizations (not shown) are at $45^{\circ}$ and $-135^{\circ}$ degrees to the $x$-axis, and there is and external field of 450 Oe applied at $85^{\circ}$ to the $x$-axis. The color scale shows the angle $\phi$ between the local magnetization and the $x$-axis.

magnetizations are approximately (these layers are also treated micromagnetically) at $45^{\circ}$ and $-135^{\circ}$ degrees to the $x$-axis, and there is an external field of magnitude 450 Oe applied in the $x y$-plane at $85^{\circ}$ to the $x$-axis (in an actual magnetic tunnel juction, there are three magnetic layers, and the reference layer is the one next to the free layer and is responsible for the spin transfer torque). The figure shows that while the magnetization is not perfectly uniform and aligned with the external field everywhere, the maximum deviation is small, about $15^{\circ}$ and localized to the edges of the magnetic disc, and an expansion in deviations from the average magnetization direction is reasonable and should converge rapidly. It is of course easy to generalize to an arbitrary local effective field direction and with a non-uniform equilibrium magnetization in the direction of the local net magnetic field, but that does introduce more variables (see Appendix A). We prefer to keep the discussion relatively simple and transparent, and also with the specific application in mind of a magnetic tunnel junction STO with an in-plane external field, or a nanocontact STO with a strong out-of-plane external field. For simplicity, we will use units in which $\gamma=1$, and because $\alpha \ll 1$ we will ignore terms of order $\alpha^{2}$. We write $\mathbf{H}_{i}=\mathbf{H}_{\mathrm{ext}}+\mathbf{H}_{d, i}$. In terms of components, the LLG 
equation is then

$$
\begin{aligned}
\frac{d m_{x, i}}{d t}= & -\left(m_{i, y} H_{i, z}-m_{i, z} H_{i, y}\right)+\alpha\left(1-m_{i, x}^{2}\right) H_{i, x}-\alpha m_{i, x}\left(m_{i, y} H_{i, y}+m_{i, z} H_{i, z}\right) \\
& -a_{J}\left(1-m_{i, x}^{2}\right) M_{x}+a_{J} m_{i, x}\left(m_{i, y} M_{y}+m_{i, z} M_{z}\right) \\
\frac{d m_{y, i}}{d t}= & -\left(m_{i, z} H_{i, x}-m_{i, x} H_{i, z}\right)+\alpha\left(1-m_{i, y}^{2}\right) H_{i, y}-\alpha m_{i, y}\left(m_{i, x} H_{i, x}+m_{i, z} H_{i, z}\right) \\
& -a_{J}\left(1-m_{i, y}^{2}\right) M_{y}+a_{J} m_{i, y}\left(m_{i, x} M_{x}+m_{i, z} M_{z}\right) \\
\frac{d m_{z, i}}{d t}= & -\left(m_{i, x} H_{i, y}-m_{i, y} H_{i, x}\right)+\alpha\left(1-m_{i, z}^{2}\right) H_{i, z}-\alpha m_{i, z}\left(m_{i, x} H_{i, x}+m_{i, y} H_{i, y}\right) \\
& -a_{J}\left(1-m_{i, z}^{2}\right) M_{z}+a_{J} m_{i, z}\left(m_{i, x} M_{x}+m_{i, y} M_{y}\right) .
\end{aligned}
$$

Next, we introduce the generalized local non-linear Holstein-Primakoff transformation of Slavin and co-workers[12, 15] that transforms the two degrees of freedom of each director $\hat{m}_{i}$ to a complex variable $c_{i}$,

$$
c_{i}=\frac{m_{y, i}-\mathrm{i} m_{z, i}}{\sqrt{2\left(1+m_{x, i}\right)}},
$$

where $\mathrm{i}$ is the imaginary unit, with the inverse transformation

$$
\hat{m}_{i}=\sqrt{1-\left|c_{i}\right|^{2}}\left[\left(c_{i}+c_{i}^{*}\right) \hat{y}+\mathrm{i}\left(c_{i}-c_{i}^{*}\right) \hat{z}\right]+\left(1-2\left|c_{i}\right|^{2}\right) \hat{x} .
$$

In terms of the local variables $c_{i}$, we can write the fields $\mathbf{H}_{d, i}$ as

$$
\begin{aligned}
& H_{d, i, x}=\sum_{i^{\prime}}\left[D_{i, i^{\prime} ; x, x}\left(1-2\left|c_{i^{\prime}}\right|^{2}\right)+D_{i, i^{\prime} ; x, y} \sqrt{1-\left|c_{i^{\prime}}\right|^{2}}\left(c_{i^{\prime}}+c_{i^{\prime}}^{*}\right)+D_{i, i^{\prime} ; x, z} \mathrm{i} \sqrt{1-\left|c_{i^{\prime}}\right|^{2}}\left(c_{i^{\prime}}-c_{i^{\prime}}^{*}\right)\right] \\
& H_{d, i, y}=\sum_{i^{\prime}}\left[D_{i, i^{\prime} ; y, x}\left(1-2\left|c_{i^{\prime}}\right|^{2}\right)+D_{i, i^{\prime} ; y, y} \sqrt{1-\left|c_{i^{\prime}}\right|^{2}}\left(c_{i^{\prime}}+c_{i^{\prime}}^{*}\right)+D_{i, i^{\prime} ; y, z} \mathrm{i} \sqrt{1-\left|c_{i^{\prime}}\right|^{2}}\left(c_{i^{\prime}}-c_{i^{\prime}}^{*}\right)\right] \\
& H_{d, i, z}=\sum_{i^{\prime}}\left[D_{i, i^{\prime} ; z, x}\left(1-2\left|c_{i^{\prime}}\right|^{2}\right)+D_{i, i^{\prime} ; z, y} \sqrt{1-\left|c_{i^{\prime}}\right|^{2}}\left(c_{i^{\prime}}+c_{i^{\prime}}^{*}\right)+D_{i, i^{\prime} ; z, z} \mathrm{i} \sqrt{1-\left|c_{i^{\prime}}\right|^{2}}\left(c_{i^{\prime}}-c_{i^{\prime}}^{*}\right)\right]
\end{aligned}
$$

By multiplying Eq. (5) by i and subtracting the result from Eq. (4), and also using $m_{x, i}=1-2\left|c_{i}\right|^{2}$ from Eq. (7) in Eq. (3), we obtain the following 
equations:

$$
\begin{aligned}
\frac{d}{d t}\left[2 \sqrt{1-\left|c_{i}\right|^{2}} c_{i}\right]= & \left(1-2\left|c_{i}\right|^{2}\right)\left(H_{i, z}+\mathrm{i} H_{i, y}\right)-2 \mathrm{i} \sqrt{1-\left|c_{i}\right|^{2}} c_{i} H_{i, x} \\
& -2 \alpha \sqrt{1-\left|c_{i}\right|^{2}} c_{i}\left(m_{i, x} H_{i, x}+m_{i, y} H_{i, y}+m_{i, z} H_{i, z}\right)+\alpha\left(H_{i, y}-i H_{i, z}\right) \\
& +2 a J \sqrt{1-\left|c_{i}\right|^{2}} c_{i}\left(m_{i, x} M_{x}+m_{i, y} M_{y}+m_{i, z} M_{z}\right)-a_{J}\left(M_{y}-i M_{\text {(女) }} 1\right) \\
-2 \frac{d}{d t}\left|c_{i}\right|^{2}= & -\sqrt{1-\left|c_{i}\right|^{2}}\left[\left(c_{i}+c_{i}^{*}\right) H_{i, z}-\mathrm{i}\left(c_{i}-c_{i}^{*}\right) H_{i, y}\right] \\
& +4 \alpha\left(1-\left|c_{i}\right|^{2}\right)\left|c_{i}\right|^{2} H_{i, x}-\alpha\left(1-2\left|c_{i}\right|^{2}\right) \sqrt{1-\left|c_{i}\right|^{2}}\left[\left(c_{i}+c_{i}^{*}\right) H_{i, y}\right. \\
& \left.+\mathrm{i}\left(c_{i}-c_{i}^{*}\right) H_{i, z}\right] \\
& -4 a_{J}\left(1-\left|c_{i}\right|^{2}\right)\left|c_{i}\right|^{2} M_{x}+a_{J}\left(1-2\left|c_{i}\right|^{2}\right) \sqrt{1-\left|c_{i}\right|^{2}}\left(c_{i}+c_{i}^{*}\right) M_{y} \\
& +\mathrm{i}\left(c_{i}-c_{i}^{*}\right) M_{z} .
\end{aligned}
$$

Finally, we combine Eqs. (11 - 12) and use

$$
\frac{d}{d t}\left[2 \sqrt{1-\left|c_{i}\right|^{2}} c_{i}\right]=2 \sqrt{1-\left|c_{i}\right|^{2}} \frac{d c_{i}}{d t}-\frac{c_{i}}{\sqrt{1-\left|c_{i}\right|^{2}}} \frac{d\left|c_{i}\right|^{2}}{d t}
$$

to obtain

$$
\begin{aligned}
\frac{d c_{i}}{d t}= & \mathrm{i} \frac{\left(H_{i, y}-i H_{i, z}\right)\left(2-3\left|c_{i}\right|^{2}\right)}{4 \sqrt{1-\left|c_{i}\right|^{2}}-\mathrm{i} H_{i, x} c_{i}-\mathrm{i} \frac{\left(H_{i, y}+i H_{i, z}\right) c_{i}^{2}}{4 \sqrt{1-\left|c_{i}\right|^{2}}}} \\
& +\alpha \frac{\left(H_{i, y}-\mathrm{i} H_{i, z}\right)\left[2-\left|c_{i}\right|^{2}\left(3-4\left|c_{i}\right|^{2}\right)\right]}{4 \sqrt{1-\left|c_{i}\right|^{2}}}-\alpha c_{i} H_{i, x}\left(1-\left|c_{i}\right|^{2}\right)-\alpha c_{i}^{2} \frac{\left(H_{i, y}+\mathrm{i} H_{i, z}\right)\left(3-4\left|c_{i}\right|^{2}\right)}{4 \sqrt{1-\left|c_{i}\right|^{2}}} \\
& -a_{J} \frac{\left(M_{y}-\mathrm{i} M_{z}\right)\left[2-\left|c_{i}\right|^{2}\left(3-4\left|c_{i}\right|^{2}\right)\right]}{4 \sqrt{1-\left|c_{i}\right|^{2}}}+a_{J} c_{i} M_{x}\left(1-\left|c_{i}\right|^{2}\right)-a_{J} c_{i}^{2} \frac{\left(M_{y}+\mathrm{i} M_{z}\right)\left(3-4\left|c_{i}\right|^{2}\right)}{4 \sqrt{1-\left|c_{i}\right|^{2}}}
\end{aligned}
$$

We also note the condition on equilibrium, which we write as

$$
\begin{aligned}
& H_{\mathrm{ext}, y}+\sum_{i^{\prime}} D_{i, i^{\prime} ; x, y}=0 \\
& H_{\mathrm{ext}, z}+\sum_{i^{\prime}} D_{i, i^{\prime} ; x, z}=0,
\end{aligned}
$$

which holds for all $i$. Equations (15 - 16) imply that the conservative and dissipative terms (containing $\alpha$ ) of order zero in $c_{i}$ in Eq. (14) vanish. Equation (14), together with Eqs. $(15$ - 16) is equivalent to the traditional LLG 
equation and describes the full non-linear magnetization motion in the presence of damping and (in-plane) STT. The advantage of this form compared to the traditional LLG form is that it is a single (complex) equation fully describing the non-linear behavior of the magnetization dynamics that allows for a systematic expansion in powers of $c_{i}$ to derive the effective timeevolution of coupled modes. For STT auto-oscillators, we must also include a non-linear dependence of the damping $\alpha$ on the oscillator power[12] - otherwise we will not get stable oscillations above threshold - and we will in general write $\alpha=\alpha_{G}\left(1+q_{1} \xi^{2}\right)$, where $\xi$ is a dimensionless measure of the oscillator energy.

\subsection{Procedure for deriving effective mode equations}

We will use Eq. (14) to analyze the effect of the non-conservative torques on an auto-oscillator, and to derive effective equations for the slow timebehavior of modes in the presence of coupling. By slow we here mean timescales much larger than characteristic resonance periods of the system, which are of the order of $0.1-1 \mathrm{~ns}$; we will make this more precise below. The strategy we will use is very similar to that used in physical optics, where Ansatz solutions are typically made for solutions that are linear combinations of eigenmodes, and the coefficients are slowly time dependent [35, 36]. To be specific, we will consider a system where two modes, labeled 1 and 2 , are dominant. This can be a system such as the one in Ref. [22] where the uniform FMR mode is most strongly excited by the spin transfer torque, but where there is mode hopping between this mode and another mode, indicative of strong couplings between those modes, or it can be a system that is close to a mode crossing, such as the system in Ref. [8]. We shall also assume that $\omega_{1}$ and $\omega_{2}$ are the two lowest frequencies in the system, and that there is a finite gap between $\omega_{i}$ and $\omega_{n}$, where $i=1,2$ and $n=3,4, \ldots$ Figure 3 depicts an experimental example of such a spectrum. Note that in the experiment in Fig. 3, there were three modes that participated in the mode hopping and as a consequence had non-thermal occupations; for simplicity, we will keep the present discussion to only two dominant modes between which there is mode hopping.

In order to express the solutions in modes, we must specify a reference system for which the modes are well defined. We will use the linearized 


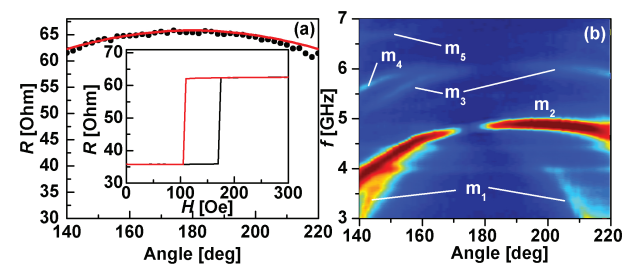

Figure 3: (Color online)(a) Experiment (circles) and calculated (solid line) resistance versus in-plane field angle $\varphi$ at $H=450$ Oe, where $\varphi=180^{\circ}$ corresponds to anti-parallel alignment of the free layer and the reference layer. The inset shows magnetoresistance loop measured at $\varphi=180^{\circ}$. (b) Map of power (dB) vs frequency and in-plane field angle for $I=8 \mathrm{~mA}$ and $H=450$ Oe showing the free layer modes. From Ref. [22] (reproduced with permission).

conservative system as a reference. This system is described by

$$
\frac{d c_{i}}{d t}=\frac{1}{2}\left(\mathrm{i} \bar{H}_{d, i, y}+\bar{H}_{d, i, z}\right)-\mathrm{i}\left[H_{\text {ext }, x}+\sum_{i^{\prime}} D_{i, i^{\prime} ; x, x}\right] c_{i} .
$$

Here, $\bar{H}_{d, i, y}$ and $\bar{H}_{d, i, z}$ are the linearized magnetostatic fields

$$
\begin{aligned}
& \bar{H}_{d, i, y}=\sum_{i^{\prime}}\left[D_{i, i^{\prime} ; y, y}\left(c_{i^{\prime}}+c_{i^{\prime}}^{*}\right)+D_{i, i^{\prime} ; y, z} \mathrm{i}\left(c_{i^{\prime}}-c_{i^{\prime}}^{*}\right)\right] \\
& \bar{H}_{d, i, z}=\sum_{i^{\prime}}\left[D_{i, i^{\prime} ; z, y}\left(c_{i^{\prime}}+c_{i^{\prime}}^{*}\right)+D_{i, i^{\prime} ; z, z} \mathrm{i}\left(c_{i^{\prime}}-c_{i^{\prime}}^{*}\right)\right] .
\end{aligned}
$$

Equation (17) describes a linear conservative system that admits a family of $N$ orthonormal (complex) eigenvectors $\left\{b_{n}\right\}, n=1,2 \ldots, N$, that span the range of the linearized equation ${ }^{1}$, with real eigenvalues $\omega_{n}$ :

$$
\begin{aligned}
\frac{d}{d t} b_{i, n}(t)=\frac{d}{d t} b_{i, n} \mathrm{e}^{-\mathrm{i} \omega_{n} t}= & -\mathrm{i} \omega_{n} b_{i, n} \mathrm{e}^{-\mathrm{i} \omega_{n} t} \\
= & \frac{\mathrm{i}}{2}\left\{\left[\sum_{i^{\prime}} D_{i, i^{\prime} ; y, y}\left(b_{i^{\prime}, n}+b_{i^{\prime}, n}^{*}\right)-2 \mathrm{i} \sum_{i^{\prime}} D_{i, i^{\prime} ; y, z} b_{i^{\prime}, n}^{*}\right]\right. \\
& \left.+\sum_{i^{\prime}} D_{i, i^{\prime} ; z, z}\left(b_{i^{\prime}, n}-b_{i^{\prime}, n}^{*}\right)\right\}-\mathrm{i}\left[H_{\text {ext }, x}+\sum_{i^{\prime}} D_{i, i^{\prime} ; x, x}\right] b_{i, n}, \\
& i=1,2 \ldots, N .
\end{aligned}
$$

\footnotetext{
${ }^{1}$ we are restricting the solution space to solutions with $\omega_{n} \geq 0$
} 
We will use short-hand notation $\left|b_{n}\right\rangle$ and $\left\langle b_{n}\right|$ for the vector $\left(b_{1, n}, b_{2, n}, \ldots, b_{N, n}\right)$ and its adjoint, respectively; the orthogonality and completeness can then be written

$$
\left\langle b_{n} \mid b_{n^{\prime}}\right\rangle=\sum_{i} b_{i, n}^{*} b_{i, n^{\prime}}=\delta_{n, n^{\prime}}
$$

and

$$
\frac{1}{2 \pi} \int_{-\infty}^{\infty} \mathrm{e}^{-\mathrm{i}\left(\omega_{n}-\omega_{n^{\prime}}\right) t} d t=\delta_{n, n^{\prime}} .
$$

The second-order terms in $c_{i}$ in Eq. (14) are

$$
\begin{aligned}
& -\frac{\mathrm{i}}{4} \sum_{i^{\prime}} D_{i, i^{\prime} ; x y}\left[4\left|c_{i^{\prime}}\right|^{2}+2\left|c_{i}\right|^{2}+4 c_{i} c_{i^{\prime}}+4 c_{i} c_{i^{\prime}}^{*}+c_{i}^{2}\right] \\
& -\frac{1}{4} \sum_{i^{\prime}} D_{i, i^{\prime} ; x z}\left[4\left|c_{i^{\prime}}\right|^{2}+2\left|c_{i}\right|^{2}-4 c_{i} c_{i^{\prime}}+4 c_{i} c_{i^{\prime}}^{*}-c_{i}^{2}\right] .
\end{aligned} .
$$

and correspond to non-resonant three-magnon processes. We can eliminate these from the equation of motion (up to third order in $c_{i}$ ) by a rotation of the basis $b_{n}$ to a basis $d_{n}$. This elimination is well-known in wave and magnon theory $[37,38,39,12]$. The transformation from $b_{n}$ to $d_{n}$ is relatively simple for translationally invariant systems, in which momentum is conserved and the equation of motion for magnons only connect magnons of momentum $\mathbf{k}$ with magnons of momentum $\pm \mathbf{k}$, and also for single-mode systems. In these cases, the transformation corresponds to a change of basis from singleoccupancy magnon states to a linear combination of empty states, single, and double-occupancy magnon states but for translationally invariant systems acts only on $(\mathbf{k},-\mathbf{k})$ sectors of the magnon Fock space. Our system is not translationally invariant and in principle a similar nonlinear transformation connects all empty, single- and double-occupancy states in the magnon Fock space. This makes the transformation a little more complicated, but it can still be explicitly constructed. We generate the transformation by first constructing a Hamiltonian from the conservative first- and second-order terms in the equations of motion, and then seeking a transformation that eliminates the second- and third-order terms from this Hamiltonian. This transformation will then in turn generate new fourth-order terms in the Hamiltonian, corresponding to third-order terms in the equation of motion.

Finally, in order to complete the analysis, we also write down the third- 
order terms in $c_{i}$ in Eq. (14) as follows

$$
\begin{aligned}
& 2 \mathrm{i} \sum_{i^{\prime}} D_{i, i^{\prime} ; x, x} c_{i}\left|c_{i^{\prime}}\right|^{2}-\frac{\mathrm{i}}{4} \sum_{i^{\prime}}\left[\left(D_{i, i^{\prime} ; y, y}+D_{i, i^{\prime} ; z, z}\right) c_{i^{\prime}}^{*}+\left(D_{i, i^{\prime} ; y, y}-D_{i, i^{\prime} ; z, z}+2 \mathrm{i} D_{i, i^{\prime} ; z, y}\right) c_{i^{\prime}}\right] c_{i}^{2} \\
& -\frac{\mathrm{i}}{4} \sum_{i^{\prime}}\left[\left(D_{i, i^{\prime} ; y, y}+D_{i, i^{\prime} ; z, z}\right) c_{i^{\prime}}+\left(D_{i, i^{\prime} ; y, y}-D_{i, i^{\prime} ; z, z}-2 \mathrm{i} D_{i, i^{\prime} ; z, y}\right) c_{i^{\prime}}^{*}\right]\left(\left|c_{i^{\prime}}\right|^{2}+2\left|c_{i}\right|^{2}\right) .
\end{aligned}
$$

As we will show in the following section, these terms correspond to the fourmagnon processes that may contribute to the linear mode coupling.

\subsubsection{Hamiltonian formulation}

The first- and second order terms in the equation of motion Eq. (14) can be generated from a Hamiltonian $H\left(c_{i}, c_{i}^{*}\right)$ through $d c_{i} /(d t)=-\mathrm{i} \partial H /\left(\partial c_{i}^{*}\right)$, where $H=\sum_{i} H_{i}$ with $H_{i}$ given by

$$
\begin{aligned}
H_{i}= & \left(H_{\mathrm{ext}, x}+\sum_{i^{\prime}} D_{i, i^{\prime} ; x, x}\right)\left|c_{i}\right|^{2}+\sum_{i^{\prime}} D_{i, i^{\prime} ; x, x}\left(\left|c_{i^{\prime}}\right|^{2}-\delta_{i, i^{\prime}} c_{i}^{*} c_{i^{\prime}}\right) \\
& -\frac{1}{2} \sum_{i^{\prime}}\left(D_{i, i^{\prime} ; y, y}+D_{i, i^{\prime} ; z, z}\right)\left(c_{i^{\prime}} c_{i}^{*}+c_{i^{\prime}}^{*} c_{i}-\delta_{i, i^{\prime}} c_{i^{\prime}}^{*} c_{i}\right) \\
& -\frac{1}{2} \sum_{i^{\prime}}\left(D_{i, i^{\prime} ; y, y}-D_{i, i^{\prime} ; z, z}\right)\left[c_{i^{\prime}}^{*} c_{i}^{*}+c_{i^{\prime}} c_{i}-\frac{1}{2} \delta_{i, i^{\prime}}\left(c_{i^{\prime}}^{*} c_{i}^{*}+c_{i^{\prime}} c_{i}\right)\right] \\
& +\mathrm{i} \sum_{i^{\prime}} D_{i, i^{\prime} ; y, z}\left[c_{i^{\prime}}^{*} c_{i}^{*}-c_{i^{\prime}} c_{i}-\frac{1}{2} \delta_{i, i^{\prime}}\left(c_{i^{\prime}}^{*} c_{i}^{*}-c_{i^{\prime}} c_{i}\right)\right] \\
& +\frac{1}{4} \sum_{i^{\prime}}\left\{\left(D_{i, i^{\prime} ; x, z}+\mathrm{i} D_{i, i^{\prime} ; y, z}\right)\left[4\left|c_{i^{\prime}}\right|^{2} c_{i}+4\left|c_{i}\right|^{2} c_{i^{\prime}}+\left|c_{i}\right|^{2} c_{i}-4 \delta_{i, i^{\prime}}\left|c_{i^{\prime}}\right|^{2} c_{i}\right]+c . c .\right\} \\
& +\sum_{i^{\prime}} D_{i, i^{\prime} ; x, x}\left(\left|c_{i^{\prime}}\right|^{4} \delta_{i, i^{\prime}}-2\left|c_{i}\right|^{2}\left|c_{i^{\prime}}\right|^{2}\right) \\
& +\frac{1}{4} \sum_{i^{\prime}}\left(D_{i, i^{\prime} ; y, y}+D_{i, i^{\prime} ; z, z}\right)\left[\left(\left|c_{i^{\prime}}\right|^{2}+\left|c_{i}\right|^{2}\right)\left(c_{i^{\prime}}^{*} c_{i}+c_{i^{\prime}} c_{i}^{*}\right)-2 \delta_{i, i^{\prime}}\left|c_{i}\right|^{4}\right] \\
& +\frac{1}{4} \sum_{i^{\prime}}\left[\left(D_{i, i^{\prime} ; y, y}-D_{i, i^{\prime} ; z, z}-2 \mathrm{i} D_{i, i^{\prime} ; z, y}\right)\left(\left|c_{i^{\prime}}\right|^{2} c_{i^{\prime}}^{*} c_{i}^{*}+\left|c_{i}\right|^{2} c_{i^{\prime}}^{*} c_{i}^{*}-\delta_{i, i^{\prime}} c_{i}^{* 2}\left|c_{i}\right|^{2}\right)+c . c .\right] .
\end{aligned}
$$


We can write Eq. (25) more compactly as

$$
\begin{aligned}
H= & \sum_{i} H_{i}=\sum_{i, i^{\prime}}\left[A_{i, i^{\prime}} c_{i}^{*} c_{i^{\prime}}+\left(B_{i, i^{\prime}} c_{i^{\prime}}^{*} c_{i}^{*}+\text { c.c. }\right)+\left(E_{i, i^{\prime}}\left|c_{i}\right|^{2} c_{i^{\prime}}^{*}+\text { c.c. }\right)\right. \\
& \left.+F_{i, i^{\prime}}\left|c_{i}\right|^{2}\left|c_{i^{\prime}}\right|^{2}+\left(G_{i, i^{\prime}} c_{i} c_{i^{\prime}}^{*}\left|c_{i^{\prime}}\right|^{2}+\text { c.c. }\right)-\left(B_{i, i^{\prime}} c_{i^{\prime}} c_{i}\left|c_{i^{\prime}}\right|^{2}+\text { c.c. }\right)\right](26
\end{aligned}
$$

where the coefficients are

$$
\begin{gathered}
A_{i, i^{\prime}}=\left(H_{\mathrm{ext}, x}-D_{i, i^{\prime} ; x, x}+2 \sum_{i, i^{\prime \prime}} D_{i, i^{\prime \prime} ; x, x}\right) \delta_{i, i^{\prime}}-\frac{1}{2}\left(D_{i, i^{\prime} ; y, y}+D_{i, i^{\prime} ; z, z}\right)\left(2-\delta_{i, i^{\prime}}\right) \\
B_{i, i^{\prime}}=-\frac{1}{4}\left(2-\delta_{i, i^{\prime}}\right)\left(D_{i, i^{\prime} ; y, y}-D_{i, i^{\prime} ; z, z}-2 \mathrm{i} D_{i, i^{\prime} ; y, z}\right) \\
E_{i, i^{\prime}}=\left(D_{i, i^{\prime} ; x, y}-\mathrm{i} D_{i, i^{\prime} ; x, z}\right)\left(2-\delta_{i, i^{\prime}}\right)+\frac{1}{4} \sum_{i^{\prime \prime}}\left(D_{i, i^{\prime \prime} ; x, y}-\mathrm{i} D_{i, i^{\prime \prime} ; x, z}\right) \delta_{i, i^{\prime}}, \\
F_{i, i^{\prime}}=-\left(2-\delta_{i, i^{\prime}}\right) D_{i, i^{\prime} ; x, x}
\end{gathered}
$$

and

$$
G_{i, i^{\prime}}=\frac{1}{4}\left(2-\delta_{i, i^{\prime}}\right)\left(D_{i, i^{\prime} ; y, y}+D_{i, i^{\prime} ; z, z}\right)
$$

\subsubsection{Elimination of non-resonant three-magnon scattering}

We seek a nonlinear transformation to a basis $\left|d_{n}\right\rangle$ that eliminates the third-order terms from the Hamiltonian Eq. (25) or (26) in the representation

of the basis $\left\{d_{n}\right\}$. In order to do so, we first perform a linear canonical transformation to diagonalize the bilinear terms of the Hamiltonian

$$
\mathcal{H}_{c}^{(2)}=\sum_{i, i^{\prime}} A_{i, i^{\prime}} c_{i}^{*} c_{i^{\prime}}+\sum_{i, i^{\prime}}\left(B_{i, i^{\prime}} c_{i^{\prime}}^{*} c_{i}^{*}+B_{i, i^{\prime}}^{*} c_{i^{\prime}} c_{i}\right)
$$

The Hamiltonian Eq. (32) can be rewritten [40] in the following matrix form

$$
\mathcal{H}_{c}^{(2)}=\frac{1}{2} \boldsymbol{\psi}^{\dagger} M \boldsymbol{\psi}
$$

The Hermitian matrix $M$ reads

$$
M=\left[\begin{array}{cc}
A & B \\
B^{\dagger} & A^{\dagger}
\end{array}\right]
$$

where $A$ and $B$ are the submatrices with entries $A_{i, i^{\prime}}$ and $B_{i, i^{\prime}}$ respectively. 
According to Eqs. (27) and (28),

$$
A^{\dagger}=A \text { and } B^{\dagger}=B^{*} \text {. }
$$

The column vector $\boldsymbol{\psi}$ and its Hermitian conjugate read

$$
\boldsymbol{\psi}=\left(\begin{array}{c}
\boldsymbol{c} \\
\boldsymbol{c}^{*}
\end{array}\right) \text { and } \boldsymbol{\psi}^{\dagger}=\left(\boldsymbol{c}^{\dagger}, \boldsymbol{c}^{T}\right)
$$

where $\mathbf{c}$ and $\mathbf{c}^{T}$ column and row vectors of size $N$ which is given by

$$
\mathbf{c}^{T}=\left(c_{1}, c_{2}, \ldots, c_{N}\right)
$$

In order to diagonalize the coefficient matrix $M$, we introduce a generalized Bogoliubov transformation

$$
\boldsymbol{c}=U \boldsymbol{b}+V \boldsymbol{b}^{*}
$$

where $\boldsymbol{b}^{T}=\left(b_{1}, b_{2}, \ldots, b_{N}\right)$, by which we construct a set of new column and row vectors of size $N$, i.e.,

$$
\boldsymbol{\varphi}=\left(\begin{array}{c}
\boldsymbol{b} \\
\boldsymbol{b}^{*}
\end{array}\right) \text { and } \boldsymbol{\varphi}^{\dagger}=\left(\boldsymbol{b}^{\dagger}, \boldsymbol{b}^{T}\right)
$$

From Eq. (38), we have

$$
\psi=T \varphi
$$

where $T$ is a so-called para-unitary matrix given by

$$
T=\left[\begin{array}{cc}
U & V \\
V^{*} & U^{*}
\end{array}\right]
$$

Note that in order for the transformation in Eq. (38) to be canonical, the matrix $T$ must satisfy the following relation

$$
T \mathfrak{I} T^{\dagger}=\mathfrak{I}
$$

where

$$
\mathfrak{I}=\left[\begin{array}{cc}
I & 0 \\
0 & -I
\end{array}\right]
$$


with $I$ being the unit matrix of dimension $N$. We seek a matrix such that Hamiltonian $\mathcal{H}^{(2)}$ is diagonal in representation of the new variable $\boldsymbol{b}$, i.e.,

$$
\mathcal{H}_{b}^{(2)}=\frac{1}{2} \varphi^{\dagger} T^{\dagger} M T \varphi
$$

with the new diagnonal coefficient matrix

$$
T^{\dagger} M T=\Lambda
$$

where $\Lambda=\operatorname{diag}\left(\lambda_{1}, \lambda_{2}, \ldots, \lambda_{2 N}\right)$ is the diagonal eigenvalue matrix. The $n^{\text {th }}$ $(n \in[1, N])$ eigenvalue and the $T$ matrix can be determined by the following secular equations

$$
\left[\begin{array}{cc}
A-\lambda_{n} I & B \\
B^{*} & A+\lambda_{n} I
\end{array}\right]\left(\begin{array}{l}
\mathbf{u}_{n} \\
\mathbf{v}_{n}^{*}
\end{array}\right)=0
$$

where $\mathbf{u}_{n}$ and $\mathbf{v}_{n}$ are the $n$-th column vectors of matrices $U$ and $V$ respectively. Furthermore, the eigenvalues can be determined by the following equation

$$
\operatorname{det}\left[\left(A-\lambda_{n} I\right)\left(A+\lambda_{n} I\right)-B^{*} B\right]=0
$$

Having found the eigenvalues, the diagonalized Hamiltonian can be rewritten as

$$
\mathcal{H}_{b}^{(2)}=\sum_{i=1}^{N}\left(\lambda_{i}+\lambda_{i+N}\right) b_{i}^{*} b_{i}
$$

By working out the above Bogoliubov transformation, the full Hamiltonian becomes

$\mathcal{H}_{b}=\sum_{i} \omega_{i} b_{i}^{*} b_{i}+\sum_{i_{1}, i_{2}, i_{3}}\left(X_{i_{1}, i_{2}, i_{3}} b_{i_{1}} b_{i_{2}} b_{i_{3}}+Y_{i_{1}, i_{2}, i_{3}} b_{i_{1}} b_{i_{2}} b_{i_{3}}^{*}+\right.$ c.c. $)+\sum_{i_{1}, i_{2}, i_{3}, i_{4}} \Gamma_{i_{1}, i_{2}, i_{3}, i_{4}} b_{i_{1}} b_{i_{2}} b_{i_{3}}^{*} b_{i_{4}}^{*}$

with $\omega_{i}=\lambda_{i}+\lambda_{i+N}$ and the coefficients

$$
\begin{aligned}
X_{i_{1}, i_{2}, i_{3}}= & \sum_{i, i^{\prime}}\left[E_{i, i^{\prime}}\left(U_{i, i_{1}} V_{i, i_{3}}^{*} V_{i^{\prime}, i_{2}}^{*}+U_{i, i_{2}} V_{i, i_{3}}^{*} V_{i^{\prime}, i_{1}}^{*}+U_{i, i_{3}} V_{i, i_{1}}^{*} V_{i^{\prime}, i_{2}}^{*}\right)\right. \\
& \left.+E_{i, i^{\prime}}^{*}\left(U_{i, i_{3}} V_{i, i_{1}}^{*} U_{i^{\prime}, i_{2}}+U_{i, i_{3}} V_{i, i_{2}}^{*} U_{i^{\prime}, i_{1}}+U_{i, i_{1}} V_{i, i_{3}}^{*} U_{i^{\prime}, i_{2}}\right)\right]
\end{aligned}
$$


and

$$
\begin{aligned}
Y_{i_{1}, i_{2}, i_{3}}= & \sum_{i, i^{\prime}}\left[E_{i, i^{\prime}}\left(U_{i, i_{1}} U_{i, i_{3}}^{*} V_{i^{\prime}, i_{2}}^{*}+U_{i, i_{1}} V_{i, i_{2}}^{*} U_{i^{\prime}, i_{3}}^{*}+V_{i, i_{3}} V_{i, i_{1}}^{*} V_{i^{\prime}, i_{2}}^{*}\right)\right. \\
& \left.+E_{i, i^{\prime}}^{*}\left(U_{i, i_{3}}^{*} U_{i, i_{1}} U_{i^{\prime}, i_{2}}+V_{i, i_{1}}^{*} V_{i, i_{3}} U_{i^{\prime}, i_{2}}+U_{i, i_{1}} V_{i, i_{2}}^{*} V_{i^{\prime}, i_{3}}\right)\right]
\end{aligned}
$$

The expression of $\Gamma_{i_{1}, i_{2}, i_{3}, i_{4}}$ is combersome and not very illuminating, so we do not present it here (see Appendix B for the full expression). Also note that we have neglected the nonresonant four-magnon terms since they do not give rise to the back-scattering processes that are responsible for the linear mode coupling in question, as we will discuss later in details.

Now we are in a position to eliminate the nonresonant three magnon terms by performing a nonlinear canonical transformation[37, 38]

$$
b_{i}=a_{i}-\sum_{i_{1}, i_{2}}\left(\frac{X_{i, i_{1}, i_{2}}^{*} a_{i_{1}}^{*} a_{i_{2}}^{*}}{\omega_{i}+\omega_{i_{1}}+\omega_{i_{2}}}+\frac{Y_{i, i_{1}, i_{2}}^{*} a_{i_{1}} a_{i_{2}}}{\omega_{i}-\omega_{i_{1}}-\omega_{i_{2}}}+\frac{2 Y_{i, i_{1}, i_{2}} a_{i_{1}} a_{i_{2}}^{*}}{\omega_{i}+\omega_{i_{2}}-\omega_{i_{1}}}\right)+O\left(a^{3}\right)
$$

There are two notes of caution that need to be kept in mind: (i) this transformation only applies to nonresonant three-magnon scattering process as the denominators of quadratic terms diverge for resonant three magnon processes; (ii) the third-order term is necessary to ensure the nonlinear transformation is canonical up to that order, which can be checked with the following Poisson brackets

$$
\sum_{i}\left(\frac{\delta b_{i^{\prime}}}{\delta a_{i}} \frac{\delta b_{i^{\prime \prime}}}{\delta a_{i}^{*}}-\frac{\delta b_{i^{\prime}}}{\delta a_{i}^{*}} \frac{\delta b_{i^{\prime \prime}}}{\delta a_{i}}\right)=0
$$

and

$$
\sum_{i}\left(\frac{\delta b_{i^{\prime}}}{\delta a_{i}} \frac{\delta b_{i^{\prime \prime}}^{*}}{\delta a_{i}^{*}}-\frac{\delta b_{i^{\prime}}}{\delta a_{i}^{*}} \frac{\delta b_{i^{\prime \prime}}^{*}}{\delta a_{i}}\right)=\delta_{i^{\prime \prime}, i^{\prime}}
$$

The full Hamiltonian now becomes

$$
\mathcal{H}_{a}=U_{0}+\sum_{i=1}^{N} \omega_{i} a_{i}^{*} a_{i}+\sum_{i_{1}, i_{2}, i_{3}, i_{4}} N_{i_{1}, i_{2}, i_{3}, i_{4}} a_{i_{1}} a_{i_{2}} a_{i_{3}}^{*} a_{i_{4}}^{*}
$$

with

$$
N_{i_{1}, i_{2}, i_{3}, i_{4}}=\Gamma_{i_{1}, i_{2}, i_{3}, i_{4}}+W_{i_{1}, i_{2}, i_{3}, i_{4}}
$$

where $\Gamma_{i_{1}, i_{2}, i_{3}, i_{4}}$ are the coefficients of the original resonant four-magnon terms and $W_{i_{1}, i_{2}, i_{3}, i_{4}}$ are coefficients of the four-magnon terms that are derived from 
the nonresonant three-magnon terms via the nonlinear canonical transformation given by Eq. (51). The general expression for $W_{i_{1}, i_{2}, i_{3}, i_{4}}$ is also rather complicated, so we shall present it in Appendix B. Here, we just point out that, physically, the resonant four-magnon processes together with the nonresonant three-magnon processes are responsible for the nonlinear spin-wave frequency shift $[41,42]$.

\subsubsection{Mode expansion}

We then expand the magnetization motion in the basis $\left\{d_{n}\right\}$ defined by the eigenvectors of the diagonalized billinear Hamiltonian given by Eq. (47), i.e.,

$$
|c(t)\rangle=\sum_{n} A_{n}(t)\left|d_{n}\right\rangle \mathrm{e}^{-\mathrm{i} \omega_{n} t},
$$

where we assume that the complex coefficients $A_{n}(t)$ have a slow time dependence, that is, the time-scale of variation of $A_{n}(t)$ is much larger than $\left(\omega_{m}\right)^{-1}$ for all $n, m=1,2, \ldots, N$. While this may be difficult to prove rigorously, it is a reasonable assumption that is also supported by experimental observations in which the time-evolution of a coupled system is slower than the periods of the eigenmodes[23, 22, 8] The exceptions are of course mode-hopping[22], or mode crossings [8] which happen on faster time scales. However, we have previously shown[28] that such events are described by adding a stochastic field to the effective equations for the coupled modes; we will not here consider mode-hopping events but are concerned with the effective equations to which stochastic fields can subsequently be added. We insert the expansion Eq. (56) in the equation for the time evolution of the magnetization, Eq. (14) and project with $\left\langle d_{n^{\prime}}\right|$. The left-hand side of the resulting equation then becomes simply $d A_{n^{\prime}}(t) /(d t) \mathrm{e}^{-\mathrm{i} \omega_{n^{\prime}} t}-\mathrm{i} \omega_{n^{\prime}} A_{n^{\prime}}(t) \mathrm{e}^{-\mathrm{i} \omega_{n^{\prime}} t}$. The second term proportional to $\omega_{n^{\prime}}$ is canceled by construction by the linear conservative part on the right-hand side of the equation. The remainder of the right-hand side is complicated, and contains projections of the non-linear conservative parts as well as the non-conservative parts on $\left\langle d_{n^{\prime}}\right|$; note that these terms will in general mix different modes $\left|d_{n}\right\rangle$ and $\left|d_{m}\right\rangle$. Rather than writing out explicitly here what the terms look like, we will instead systematically analyze terms of different order in $c_{i}$.

The general procedure we will follow is to expand all $c_{i}$ 's in the basis $\left|d_{n}\right\rangle$, and then multiply both sides of the equation with $\mathrm{e}^{\mathrm{i} \omega_{n^{\prime}} t}$ and integrate over time. The crucial assumption now is that the time dependence of $A_{n}(t)$ is sufficiently slow that it can be held constant during the time integration 
and moved outside of the integral, while for the integration over exponential factor, we can use Eq. (22). This means we can also ignore correlations between $A_{n}(t)$ and $A_{n^{\prime}}(t)$ on times of the order of $\omega_{m}^{-1}$ for all $n, n^{\prime}, m$ while the projected solutions onto modes 1 and 2 will couple $A_{1}(t)$ and $A_{2}(t)$ with temporal correlations on time scales much larger than $\omega_{m}^{-1}$ for all $m$. Each time-integration will then give rise to a condition on the frequency components. These kinds of terms with the condition on the frequencies are entirely analogous to a magnon-magnon scattering vertex, with the condition on the frequencies corresponding to energy conservation at the vertex. In spin wave Hamiltonian theory, the Holstein-Primakoff transformation is usually applied, and a subsequent expansion of $\sqrt{1-\hat{n}_{i}}$, where $\hat{n}_{i}$ is the (bosonic) spin number operator at site $i$, leads to magnon interactions in which magnons scatter off each other. Spin torque oscillators typically have large enough amplitudes that nonlinear processes are important and have to be included. Therefore, the expansions in $\left|c_{i}\right|$ and subsequent expansions of $c_{i}$ in eigenmode amplitudes $\left|A_{n}\right|$ have to go beyond linear terms, and processes that involve more than two quanta of spin waves have to be considered. This is how linear terms like $d A_{1} / d t \propto A_{2}(t)$ can arise as nonlinear processes that include a bath of magnons give some flexibility in satisfying energy conservation in the scattering processes. We shall here expand only up to third order although we will, for some special cases, discuss fourth-order contributions that enter because of the terms in $a_{J} \sin \beta$, as well some higher-order contributions.

Finally, we will assume that all modes $n$ other than modes 1 and 2 are in thermal equilibrium and their populations can be described by an equilibrium Bose-Einstein distribution function $n_{B}\left(\omega_{n}\right)$. This is consistent with experiments[22] in which a dominant mode is observed, in which the system spends most of its time, and a small number, e.g., one or two, of other modes that is coupled to the dominant one with mode-hopping observed between them. This implies that we are assuming that scattering events between modes 1 or 2 and other modes $m, n \neq 1,2$ are infrequent compared to the equilibration time of modes $m, n$.

\section{Results and Discussion}

\subsection{Non-conservative torques}

We will first use Eq. (14) to analyze the effect of the non-conservative torques on an auto-oscillator. By assumption, the magnetization motion 
is oscillatory with a period $\tau$, and the magnetization amplitude remains constant or invariant under long times. The dissipation of the system is described by the time-rate of change of the oscillator power, proportional to $\sum_{i}\left|c_{i}\right|^{2}$, averaged over a period $\tau$. For simplicity, and ease of notation, we now use a macro-spin model[43] with a single amplitude $c$. The time-rate of change of $|c|^{2}$ is then given by

$$
\begin{aligned}
\frac{d}{d t}|c|^{2}= & \sqrt{1-|c|^{2}}\left[\Re(c) H_{z}+\Im(c) H_{y}\right] \\
& +\frac{1}{\sqrt{1-|c|^{2}}}\left\{\left[\Re(c)\left(\alpha H_{y}-a_{J} M_{y}\right)-\Im(c)\left(\alpha H_{z}-a_{J} M_{z}\right)\right]\left[1+|c|^{2}\left(-3+2|c|^{2}\right)\right]\right\} \\
& -2\left(1-|c|^{2}\right)|c|^{2}\left[\alpha H_{x}-a_{J} M_{x}\right] .
\end{aligned}
$$

Here $\Re(c)$ and $\Im(c)$ are the real and imaginary parts of $c$, respectively. We note that Eq. (57) expresses the instantaneous $d|c|^{2} /(d t)$ which includes the transverse effective field; this can at any instant pump energy in or out of the system, but the average over one period will be zero. Under the assumption that $\hat{x}$ and $\hat{z}$ are in the $\hat{x}^{\prime} \hat{z}^{\prime}$-plane we can write $H_{d, y}=-N_{y} M_{S} m_{y}=$ $-2 N_{y} M_{S} \sqrt{1-|c|^{2}} \Re(c), H_{d, x}=-N_{x} M_{S} m_{x}+D_{x z} m_{z}=-N_{x} M_{S}\left(1-2|c|^{2}\right)-$ $2 D_{x z} \sqrt{1-|c|^{2}} \Im(c)$, and $H_{d, z}=-N_{z} M_{S} m_{z}+D_{x z} m_{x}=2 N_{z} M_{S} \sqrt{1-|c|^{2}} \Im(c)+$ $D_{x z}\left(1-2|c|^{2}\right)$, with $N_{x}, N_{y}, N_{z}$, and $D_{x z}$ real numbers. Then $\frac{1}{\sqrt{1-|c|^{2}}} \Re(c) H_{d, y}=$ $-2 N_{y} M_{S}[\Re(c)]^{2}$ and $\frac{1}{\sqrt{1-|c|^{2}}} \Im(c) H_{d, z}=2 N_{z} M_{S}[\Im(c)]^{2}+\frac{D_{x z} \Im(c)}{\sqrt{1-|c|^{2}}}\left(1-2|c|^{2}\right)$. We demand that the dissipation averaged over a period $\tau$ is zero, so that

$$
\left\langle\frac{d}{d t}|c|^{2}\right\rangle=\frac{1}{\tau} \int_{t}^{t+\tau} \frac{d}{d t}\left|c\left(t^{\prime}\right)\right|^{2} d t^{\prime}=0 .
$$

For an oscillatory motion we can assume

$$
\frac{1}{\tau} \int_{t}^{t+\tau}\left[\Re\left(c\left(t^{\prime}\right)\right)\right]^{2} d t^{\prime}=\bar{c}^{2}>0,
$$

which defines the number $\bar{c}$. The motion will in general be eccentric with eccentricity $\epsilon$, so

$$
\frac{1}{\tau} \int_{t}^{t+\tau}\left[\Im\left(c\left(t^{\prime}\right)\right)\right]^{2} d t^{\prime} \approx \epsilon \bar{c}^{2} .
$$

Futhermore, for oscillatory motion we have

$$
\frac{1}{\tau} \int_{t}^{t+\tau} \Re\left[c\left(t^{\prime}\right)\right] d t^{\prime} \approx 0 \approx \frac{1}{\tau} \int_{t}^{t+\tau} \Re\left[c\left(t^{\prime}\right)\right] \Im\left[c\left(t^{\prime}\right)\right] d t^{\prime} .
$$


To $\mathcal{O}\left(c^{2}\right)$ we then obtain

$$
\left\langle\frac{d}{d t}|c|^{2}\right\rangle=-M_{S}\left(N_{y}+\epsilon N_{z}\right) \alpha_{G}-\left[\alpha_{G}\left(H_{\mathrm{ext}, x}-N_{x} M_{S}\right)-a_{J} M_{x}\right][1+\epsilon]=0,
$$

where a factor of $\bar{c}^{2}$ cancels out. This analysis allows us to draw three conclusions: $(i)$ the time-dependent demagnetizing fields enhance the average dissipation[44] by a factor of $M_{S}\left(N_{y}+\epsilon N_{z}\right) \alpha$; (ii) only the average dissipation is zero, but not the instantaneous dissipation, and during some fraction of a period net energy is pumped into the system, and during some other fraction of a period net energy is dissipated[22] (this is also why the instantaneous value of $d|c|^{2} /(d t)$ does not equal the rate of dissipation, as $d|c|^{2} /(d t)$ fluctuates during one period of the motion); (iii) as the angle between the reference layer magnetization $\hat{M}$ and the in-plane equilibrium magnetization increases towards $\pi / 2$ the pumping through STT becomes less and less effective to offset dissipative losses and it becomes in general impossible to obtain self-sustained auto-oscillations. The threshold current is the current at which the average dissipation is zero. From Eq. (62) we see that the spin torque effective field $a_{J}$, which is proportional to the current, and $M_{x}$ enters as a product. This implies that the threshold current increases as $1 / M_{x}$ as the equilibrium magnetization direction is rotated away from the direction of the reference layer. An increase in threshold current with angle has indeed been observed experimentally $[45,22]$. The dependence on the product $a_{J} M_{x}$ also implies an invariance: if a decrease in $M_{x}$ is offset by an increase in $a_{J}$ by increasing the current such that the product $a_{J} M_{x}$ is constant the system is invariant. This is not consistent with experimental observations, where, for example, mode-hopping increases dramatically as $M_{x}$ is decreased[22]. This can only be caused by the apperance of terms in $a_{J} M_{y}$ in the coupled-mode equations. $M_{y} \neq 0$ also opens up new scattering channels between modes, which increases mode-mode scattering and the rate of mode hopping.

\subsection{Elliptical devices, in-plane field}

In this subsection, we will discuss the equations of motion for nanoscale patterned elliptical devices subjected to an applied in-plane magnetic field. This applies, for example, to the experiments in Ref. [22] in which modehopping in circular MTJ devices was investigated as a function of in-plane field angle and current. We will also for simplicity assume that the demagnetizing tensor in this case is diagonal; this is not an unreasonable approxima-

tion for elliptical planar systems. With the external field in-plane, we take 
its direction as well as the equilibrium magnetization direction in the free layer to be the $x$-axis, and introduce an angle $\beta$ between the fixed layer magnetization direction and the $x$-axis. We can then write the general equation of motion for the expansion coefficients $c_{i}$ as

$$
\begin{aligned}
\frac{d c_{i}}{d t}= & \mathrm{i} \frac{\left(H_{d, i, y}-\mathrm{i} H_{d, i, z}\right)\left(2-3\left|c_{i}\right|^{2}\right)}{4 \sqrt{1-\left|c_{i}\right|^{2}}}-\mathrm{i}\left(H_{\text {ext }}+H_{d, i, x}\right) c_{i}-\frac{\left(\mathrm{i} H_{d, i, y}-H_{d, i, z}\right) c_{i}^{2}}{4 \sqrt{1-\left|c_{i}\right|^{2}}} \\
& +\alpha \frac{\left(H_{d, i, y}-\mathrm{i} H_{d, i, z}\right)\left[2-\left|c_{i}\right|^{2}\left(3-4\left|c_{i}\right|^{2}\right)\right]}{4 \sqrt{1-\left|c_{i}\right|^{2}}} \\
& -\alpha c_{i}\left(H_{\text {ext }}+H_{d, i, x}\right)\left(1-\left|c_{i}\right|^{2}\right)-\alpha c_{i}^{2} \frac{\left(H_{d, i, y}+\mathrm{i} H_{d, i, z}\right)\left(3-4\left|c_{i}\right|^{2}\right)}{4 \sqrt{1-\left|c_{i}\right|^{2}}} \\
& -a_{J} \frac{\left[2-\left|c_{i}\right|^{2}\left(3-4\left|c_{i}\right|^{2}\right)\right] \sin [\beta]}{4 \sqrt{1-\left|c_{i}\right|^{2}}}+a_{J} c_{i}\left(1-\left|c_{i}\right|^{2}\right) \cos [\beta]-a_{J} c_{i}^{2} \frac{\left(3-2\left|c_{i}\right|^{2}\right) \sin [\beta]}{4 \sqrt{1-\left|c_{i}\right|^{2}}}
\end{aligned}
$$

We note that in this case there are no second-order conservative terms because of the diagonal demagnetizing tensor. Therefore, there are in this case no non-resonant three-magnon processes that need to be elimininated by a canonical transformation, and the bases $\left|b_{n}\right\rangle$ and $\left|d_{n}\right\rangle$ are identical.

\subsubsection{Linear non-conservative terms}

The linear non-conservative term on the right-hand side of Eq. (63) is

$$
\frac{1}{2} \alpha_{G}\left(\bar{H}_{d, i, y}-\mathrm{i} \bar{H}_{d, i, z}\right)-c_{i}\left[\alpha_{G}\left(H_{\mathrm{ext}}+\sum_{i^{\prime}} D_{i, i^{\prime} ; x, x}\right)-a_{J} M \cos [\beta]\right],
$$

and describes the first-order damping (terms in $\alpha_{G}$ ) and pumping (term in $\left.a_{J}\right)$. Expanding in eigenmodes, projecting with $\left\langle d_{n^{\prime}}\right|$, multiplying by $\mathrm{e}^{-\mathrm{i} \omega_{n^{\prime}} t}$ and integrating over time simply leaves

$$
\left(-\alpha_{G} \omega_{n^{\prime}}+a_{J} M \cos [\beta]\right) A_{n^{\prime}}(t) .
$$

As expected, to first order the damping (given by $\alpha_{G}$ ) is offset by the effective pumping (given by $a_{J} M_{x}$ ). We introduce $\sigma_{0}$ through $a_{J}=\sigma_{0} I$, where $I$ is the total current in the system, and for $n^{\prime}=1$ we can define $\Gamma_{G, 1}=\alpha_{G} \omega_{1}$. We can then write Eq. (65) in the form $[15]-\left[\Gamma_{G, 1}-\sigma_{0} I\right] A_{1}(t)$. In a system with two dominant modes, the linearized non-conservative terms for mode 2 will be analogous, but with $\Gamma_{G, 1}$ replaced by $\Gamma_{G, 2}=\alpha_{G} \omega_{2}$. 

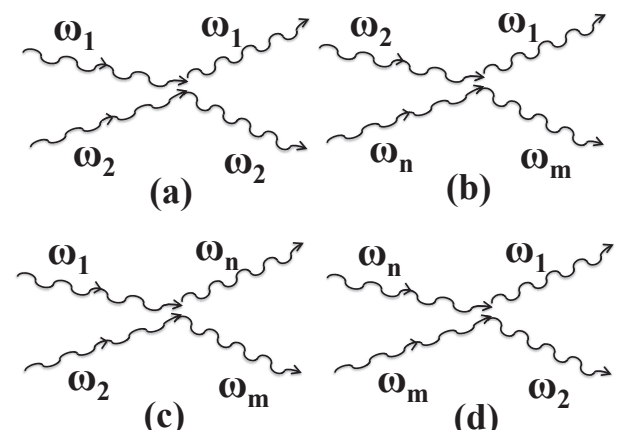

Figure 4: Depiction of scattering events coupling modes 1 and 2.

\subsubsection{Cubic conservative terms}

The cubic conservative terms on the right-hand side of Eq. (14) are

$$
\begin{aligned}
& -\frac{\mathrm{i}}{4} \sum_{i^{\prime}}\left\{D_{i, i^{\prime} ; y, y}\left[\left(c_{i^{\prime}}+c_{i^{\prime}}^{*}\right)\left(\left|c_{i^{\prime}}\right|^{2}+2\left|c_{i}\right|^{2}+c_{i}^{2}\right)\right]+D_{i, i^{\prime} ; z, z}\left[\left(c_{i^{\prime}}-c_{i^{\prime}}^{*}\right)\left(\left|c_{i^{\prime}}\right|^{2}+2\left|c_{i}\right|^{2}-c_{i}^{2}\right]\right\}\right. \\
& +2 \mathrm{i} c_{i} \sum_{i^{\prime}} D_{i, i^{\prime} ; x, x}\left|c_{i^{\prime}}\right|^{2}
\end{aligned}
$$

We insert the expansion $c_{i^{\prime}}=\sum_{n} A_{n}(t) d_{i^{\prime}, n} \mathrm{e}^{-\mathrm{i} \omega_{n} t}$, multiply on the left by $d_{i, n^{\prime}}^{*} \mathrm{e}^{\mathrm{i} \omega_{n^{\prime}} t}$, sum over $i$ and integrate over $t$ [ignoring the slow time dependence of $A(t)$ ]. The cubic terms give rise to different possibilities of mode combinations. The terms in $\left|c_{i}\right|^{2}$ and $\left|c_{i^{\prime}}\right|^{2}$ give rise to terms like

$$
\sum_{n, m, m^{\prime}} A_{n} A_{m} A_{m^{\prime}}^{*} \mathrm{e}^{-\mathrm{i}\left(\omega_{n}+\omega_{m}-\omega_{m^{\prime}}-\omega_{n^{\prime}}\right) t} \pm \sum_{n, m, m^{\prime}} A_{n}^{*} A_{m} A_{m^{\prime}}^{*} \mathrm{e}^{-\mathrm{i}\left(-\omega_{n}+\omega_{m}-\omega_{m^{\prime}}-\omega_{n^{\prime}}\right) t}
$$

which give non-zero contributions if $\omega_{n}+\omega_{m}-\omega_{m^{\prime}}-\omega_{n^{\prime}}=0$ for the first sum, or $-\omega_{n}+\omega_{m}-\omega_{m^{\prime}}-\omega_{n^{\prime}}=0$ for the second one. The different possibilities coupling modes 1 and 2 according to the first sum are depicted in Fig. 4. The special case $n=n^{\prime}=m=m^{\prime}=1$ gives the non-linear frequency shift for a single-mode theory (not depicted in Fig. 4). For a two-mode theory with $n^{\prime}=m=1$ and $n=m^{\prime}=2$ we obtain the off-diagonal non-linear frequency shift of Muduli, Heinonen, and Åkerman[22] [Fig. 4 (a)]. Scattering processes such as the one depicted in Fig. 4 (b) will give rise to linear terms of the form 
$d A_{1}(t) / d t \propto B A_{2}(t)$, with $B$ some constant, provided $\omega_{1}+\omega_{m}=\omega_{2}+\omega_{n}$, or stated differently, provided $\omega_{2}-\omega_{1}=\omega_{m}-\omega_{n}$, can be satisfied, and (ii) mode $n$ is occupied. The latter condition is generally satisfied at finite temperatures due to thermal occupation. The former can in general be satisfied because firstly, the magnon energies are small compared to room temperature or elevated temperatures in the STOs because of Joule heating. Therefore, modes in a very large portion of the magnon spectrum are thermally occupied. Secondly, there will then be magnon modes $m$ and $n$ somewhere in the thermally occupied part of the spectrum such that the energy conservation requirement $\omega_{2}-\omega_{1}=\omega_{m}-\omega_{n}$ can be satisfied as the spacing between magnon frequencies is not uniform. The possible contributions to this kind of linear coupling also increase with increasing order in $c_{i}$. For example, in fifth order incoming modes $\omega_{2}$ and $\omega_{n}$ can scatter into modes $\omega_{1}, \omega_{m}, \omega_{m^{\prime}}$ and $\omega_{m^{\prime \prime}}$, provided $\omega_{2}+\omega_{n}=\omega_{1}+\omega_{m}+\omega_{m^{\prime}}+\omega_{m^{\prime \prime}}$. The contributions to this linear coupling also increases as a mode crossing is approached so that $\omega_{1} \rightarrow \omega_{2}$, in which case $\omega_{m} \rightarrow \omega_{n}$ and energy conservation is satisfied for any mode $\omega_{n}$. Other possible scattering events coupling modes 1 and 2 are depicted in Fig. 4 (c) and (d), but the contributions from such events are zero because under the assumptions that modes 1 and 2 have the lowest frequencies, these events cannot satisfy energy conservation at the vertex. Similarly, the second sum in Eq. (67) correspond to one incoming mode and three outgoing modes at the vertex for which energy cannot be conserved at the vertex with $n^{\prime}=1$ (2) and $n=2(1)$.

The terms in $c_{i}^{2}$ in Eq. (66) give rise to contributions of the form

$$
\sum_{n, m, m^{\prime}} A_{n} A_{m} A_{m^{\prime}} \mathrm{e}^{-\mathrm{i}\left(\omega_{n}+\omega_{m}+\omega_{m^{\prime}}-\omega_{n^{\prime}}\right) t} \pm \sum_{n, m, m^{\prime}} A_{n}^{*} A_{m} A_{m^{\prime}} \mathrm{e}^{-\mathrm{i}\left(-\omega_{n}+\omega_{m}+\omega_{m^{\prime}}-\omega_{n^{\prime}}\right) t},
$$

which, after integrating over time, will give rise to non-zero terms provided $\omega_{n}+\omega_{m}+\omega_{m^{\prime}}-\omega_{n^{\prime}}=0$, or $-\omega_{n}+\omega_{m}+\omega_{m^{\prime}}-\omega_{n^{\prime}}=0$. The former cannot be satisfied for events coupling modes 1 and 2 since there are three incoming modes, two of which have higher frequencies than modes 1 or 2 . The second sum in Eq. (68) gives rise to the same kind of terms as the first sum in Eq. (67).

For an effective two-mode theory with modes 1 and 2 dominant, the 
diagonal and off-diagonal nonlinear frequency shifts of mode 1 are given by

$$
\begin{aligned}
& -\mathrm{i} A_{1}\left|A_{1}\right|^{2} \sum_{i, i^{\prime}}\left(D_{i, i^{\prime} ; y, y}+D_{i, i^{\prime} ; z, z}\right) \Re\left[d_{i, 1}^{*} d_{i^{\prime}, 1}\left|d_{i^{\prime}, 1}\right|^{2}\right] \\
& +2 \mathrm{i} A_{1}\left|A_{1}\right|^{2} \sum_{i, i^{\prime}} D_{i, i^{\prime} ; x, x}\left|d_{i, 1}\right|^{2}\left|d_{i^{\prime}, 1}\right|^{2} \\
& -\mathrm{i} A_{1}\left|A_{2}\right|^{2} \sum_{i, i^{\prime}}\left[D_{i, i^{\prime} ; y, y}+D_{i, i^{\prime} ; z, z} \Re\left[d_{i, 1} d_{i^{\prime}, 1}^{*}\left|d_{i^{\prime}, 2}\right|^{2}+\left|d_{i, 1}\right|^{2} d_{i, 2} d_{i^{\prime}, 2}^{*}\right]\right. \\
& +2 \mathrm{i} A_{1}\left|A_{2}\right|^{2} \sum_{i, i^{\prime}} D_{i, i^{\prime} ; x, x}\left[\left|d_{i, 1}\right|^{2}\left|d_{i^{\prime}, 2}\right|^{2}+d_{i, 1}^{*} d_{i^{\prime}, 1} d_{i^{\prime}, 2}^{*} d_{i, 2}\right],
\end{aligned}
$$

where we have used the symmetry $D_{i, i^{\prime} ; y, y}=D_{i^{\prime}, i ; y, y}$ and $D_{i, i^{\prime} ; z, z}=D_{i^{\prime}, i ; z, z}$. This also assures that the total contribution in Eq. (69) is imaginary and so represents a nonlinear frequency shift and no dissipation.

The other modes $n$ and $m$ constitute a bath of thermally populated modes. With modes 1 and 2 dominant the resonant terms must satisfy $\omega_{n}+\omega_{2}-\omega_{m}-\omega_{1}=0$, which give rise to "backscattering" terms:

$$
\begin{aligned}
\frac{d A_{1}(t)}{d t}= & -\frac{\mathrm{i}}{4} A_{2} \sum_{i, i^{\prime}, m, n} d_{i, 1}^{*} d_{i^{\prime}, n}\left[d_{i^{\prime}, m}^{*} d_{i^{\prime}, 2}+2 d_{i, m}^{*} d_{i^{\prime}, 2}\right]\left[D_{i, i^{\prime} ; y, y}+D_{i, i^{\prime} ; z, z}\right] \\
& \times\left[1+n_{B}\left(\omega_{m}\right)\right] n_{B}\left(\omega_{n}\right) \delta(2+n-m-1),
\end{aligned}
$$

where we have used the assumed thermal equilibrium of modes $m$ and $n$ to replace $A_{n}$ and $A_{m}^{*}$ with $n_{B}\left(\omega_{n}\right)$ and unity, respectively, by taking a thermal average over magnon states. The energy-conserving delta-function $\delta(2+$ $n-m-1)$ is short-hand for $\delta\left(\omega_{2}+\omega_{n}-\omega_{m}-\omega_{1}\right)$. We note that both the nonlinear frequency shift as well as the linear "backscattering" term are driven by non-local nonlinear interactions inherent in the LLG equation.

\subsubsection{Cubic non-conservative terms}

Just like for the conservative terms, the expansion of $1 / \sqrt{1-\left|c_{i}\right|^{2}}$ in the denominator and $\sqrt{1-\left|c_{i^{\prime}}\right|^{2}}$ in the field terms $H_{d, i^{\prime}, y}$ and $H_{d . i^{\prime}, z}$, together with the factors $\left|c_{i}\right|^{2}$ and $c_{i}^{2}$ will give rise to four-magnon vertex terms of the same structures as the conservative third-order terms and under the same conditions on the magnon frequencies. As these terms arise from the non-conservative torques, they will give rise to non-linear damping and pumping with both diagonal terms, as in the single-mode theory, and offdiagonal terms as in the two-mode description by Mudulu, Heinonen, and 
Akerman[22]. Finally, there will also in an effective two-mode theory be a linear contribution of the form $d A_{1}(t) / d t \propto A_{2}(t)$ if there exist modes $m, n$ such that $\omega_{n}+\omega_{2}-\omega_{m}-\omega_{1}=0$. Again, this term can contribute only when there is a bath of thermally excited modes $n$.

With $\alpha=\alpha_{G}\left[1+q_{1} \xi^{2}\right]$, the total cubic non-conservative terms are

$$
\begin{aligned}
& -\frac{\alpha_{G}}{4} \sum_{i^{\prime}} D_{i, i^{\prime} ; y, y}\left(c_{i^{\prime}}+c_{i^{\prime}}^{*}\right)\left[3 c_{i}^{2}+2\left|c_{i}\right|^{2}+\left|c_{i^{\prime}}\right|^{2}\right] \\
& -\frac{\alpha_{G}}{4} \sum_{i^{\prime}} D_{i, i^{\prime} ; z, z}\left(c_{i^{\prime}}-c_{i^{\prime}}^{*}\right)\left[-3 c_{i}^{2}+2\left|c_{i}\right|^{2}+\left|c_{i^{\prime}}\right|^{2}\right] \\
& +\alpha_{G} c_{i}\left|c_{i}\right|^{2}\left[H_{\mathrm{ext}}+H_{d, i, x}^{0}\right]+2 \alpha_{G} c_{i} \sum_{i^{\prime}} D_{i, i^{\prime} ; x, x}\left|c_{i^{\prime}}\right|^{2}-a_{J} c_{i}\left|c_{i}\right|^{2} \cos \beta \\
& +\frac{q_{1} \alpha_{G}}{2} \xi^{2} \sum_{i^{\prime}}\left[\left(c_{i^{\prime}}+c_{i^{\prime}}^{*}\right) D_{i, i^{\prime} ; y, y}+\left(c_{i^{\prime}}-c_{i^{\prime}}^{*}\right) D_{i, i^{\prime} ; z, z}\right] \\
& -q_{1} \xi^{2} \alpha_{G} c_{i}\left(H_{\mathrm{ext}}-H_{d, i, x}^{0}\right),
\end{aligned}
$$

where $H_{d, i, x}^{0}$ is the zero-th order demagnetizing field, $H_{d, i, x}^{0}=\sum_{i^{\prime}} D_{i, i^{\prime} ; x, x}$. Again, for two dominant modes 1 and 2 we take $\xi^{2}=\left|A_{1}\right|^{2}+\left|A_{2}\right|^{2}$. We consider resonant scattering corresponding to Fig. 4 (a), and get the following contributions to the diagonal and off-diagonal non-linear damping and 
pumping:

$$
\begin{aligned}
& -\frac{\alpha_{G}}{2} A_{1}\left|A_{1}\right|^{2} \sum_{i, i^{\prime}}\left[D_{i, i^{\prime} ; y, y}+D_{i, i^{\prime} ; z, z}\right]\left[2\left|d_{i, 1}\right|^{2} d_{i, 1} d_{i^{\prime}, 1}^{*}+\left|d_{i, 1}\right|^{2} d_{i, 1}^{*} d_{i^{\prime}, 1}\right] \\
& +\alpha_{G} A_{1}\left|A_{1}\right|^{2} \sum_{i}\left[H_{\mathrm{ext}}+H_{d, i, x}^{0}\right]\left|d_{i, 1}\right|^{2}\left|d_{i, 1}\right|^{2}+2 \alpha_{G} A_{1}\left|A_{1}\right|^{2} \sum_{i, i^{\prime}} D_{i, i^{\prime} ; x, x}\left|d_{i, 1}\right|^{2}\left|d_{i^{\prime}, 1}\right|^{2} \\
& -a_{J} A_{1}\left|A_{1}\right|^{2} \cos \beta \sum_{i}\left|d_{i, 1}\right|^{2}\left|d_{i, 1}\right|^{2} \\
& +\frac{q_{1} \alpha_{G}}{2} A_{1}\left[\left|A_{1}\right|^{2}+\left|A_{2}\right|^{2}\right] \sum_{i, i^{\prime}}\left[D_{i, i^{\prime} ; y, y}+D_{i, i^{\prime} ; z, z}\right] d_{i, 1}^{*} d_{i^{\prime}, 1} \\
& -q_{1} \alpha_{G} A_{1}\left[\left|A_{1}\right|^{2}+\left|A_{2}\right|^{2}\right] \sum_{i}\left[H_{\mathrm{ext}}-H_{d, i, x}^{0}\right]\left|d_{i, 1}\right|^{2} \\
& -\alpha_{G} A_{1}\left|A_{2}\right|^{2} \sum_{i, i^{\prime}}\left[D_{i, i^{\prime} ; y, y}+D_{i, i^{\prime} ; z, z}\right]\left[\left|d_{i, 1}\right|^{2} d_{i, 2} d_{i^{\prime}, 2}^{*}+\Re\left(\left|d_{i, 1}\right|^{2} d_{i^{\prime}, 2}^{*} d_{i, 2}+d_{i, 1}^{*} d_{i^{\prime}, 1}\left|d_{i^{\prime}, 2}\right|^{2}\right)\right] \\
& +2 \alpha_{G} A_{1}\left|A_{2}\right|^{2} \sum_{i}\left[H_{\mathrm{ext}}+H_{d, i, x}^{0}\right]\left|d_{i, 1}\right|^{2}\left|d_{i, 2}\right|^{2} \\
& +2 \alpha_{G} A_{1}\left|A_{2}\right|^{2} \sum_{i, i^{\prime}} D_{i, i^{\prime} ; x, x}\left[\left|d_{i, 1}\right|^{2}\left|d_{i^{\prime}, 2}\right|^{2}+d_{i, 1}^{*} d_{i^{\prime}, 1} d_{i, 2} d_{i^{\prime}, 2}^{*}\right] \\
& -2 a_{J} A_{1}\left|A_{2}\right|^{2} \cos \beta \sum_{i}\left|d_{i, 1}\right|^{2}\left|d_{i, 2}\right|^{2} .
\end{aligned}
$$

Note that the contribution from Eq. (72) is real (because of the symmetry $D_{i, i^{\prime} ; y, y}=D_{i^{\prime}, i ; y, y}$ and $\left.D_{i, i^{\prime} ; z, z}=D_{i^{\prime}, i ; z, z}\right)$ so it represents damping or pumping.

By considering scattering with thermally populated modes, as outlined earlier, we can also generate contributions to the "backscattering" terms analogous to Eq. (70). Again, considering modes 1 and modes 2 the dominant modes we obtain a contribution

$$
\begin{aligned}
& \frac{d A_{1}(t)}{d t}=-\frac{\alpha_{G}}{4} A_{2} \sum_{i, i^{\prime}, m, n}\left[D_{i, i^{\prime} ; y, y}+D_{i, i^{\prime} ; z, z}\right] \\
\times \quad & {\left[2 d_{i, 1}^{*} d_{i^{\prime}, 2} d_{i, m}^{*} d_{i, n}+2 d_{i, 1}^{*} d_{i, 2} d_{i, m}^{*} d_{i^{\prime}, n}+d_{i, 1}^{*} d_{i^{\prime}, 2} d_{i^{\prime}, m}^{*} d_{i^{\prime}, n}+3 d_{i, 1}^{*} d_{i, 2} d_{i^{\prime}, m}^{*} d_{i, n}\right] } \\
& \times\left[1+n_{B}\left(\omega_{m}\right)\right] n_{B}\left(\omega_{n}\right) \delta\left(\omega_{2}+\omega_{n}-\omega_{m}-\omega_{1}\right) \\
+ & A_{2} \sum_{i, i^{\prime}, m, n}\left[\frac{\alpha_{G}}{4}\left(H_{\mathrm{ext}}+H_{d, i, x}^{0}\right)-a_{J} \cos [\beta]\right] d_{i, 1}^{*} d_{i, 2} d_{i, m}^{*} d_{i, n} \\
& \times\left[1+n_{B}\left(\omega_{m}\right)\right] n_{B}\left(\omega_{n}\right) \delta(n+2-m-1) .
\end{aligned}
$$


This equation in general describes energy transfer between modes 1 and 2 because it arises from four-magnon scattering events where a mode 2 magnon is annihilated and a mode 1 magnon is created with the energy balance taken from the thermal magnons modes $m$ and $n$. This also implies that when the thermal modes are integrated out, there is a net transfer of energy in or out of modes 1 and 2. Therefore, in order to described the system in terms of the restricted space of only modes 1 and 2, some energy-conserving constraint will have to be imposed as in Refs. [[22, 25, 30]].

\subsection{4. $\sin \beta \neq 0$}

For $\sin \beta \neq 0$, that is, when the fixed layer direction is not aligned with the equilibrium magnetization direction of the free layer, there arise new terms of different symmetry than what is otherwise the case: the terms in $\sin \beta$ are all in even powers of $\left|c_{i}\right|$ or $c_{i}$. This is in contrast with the other terms (both conservative and non-conservative ones), that all have odd powers in $c_{i}$ or $\left|c_{i^{\prime}}\right|$. Therefore, these terms in $\sin \beta$ cannot be canceled by the other conservative or non-conservative terms. As a consequence, new mode-mode scattering channels open up when the applied external field is rotated away from the direction of the fixed layer magnetization. Again, considering the two dominant modes 1 and 2, the lowest-order contributions from the terms in $c_{i}^{2}$ coupling modes 1 and 2 occur if $\omega_{2}+\omega_{m}=\omega_{1}$, or $\omega_{1}+\omega_{m}=\omega_{2}$, or $\omega_{1}+\omega_{2}=\omega_{m}$ is satisfied for some $m$. The first two of these are not allowed as $\omega_{m}>\omega_{2}, \omega_{1}$, but the last represents the scattering of a magnon pair of modes 1 and 2 into mode $m$. Similarly the lowest-order term in $\left|c_{i}\right|^{2}$ only gives a non-zero contributions if $\omega_{m}=\omega_{2}+\omega_{1}$, which represents a decay of a thermally populated mode $m$ into modes 1 and 2 . The available phase-space that satisfies the requirement $\omega_{m}=\omega_{2}+\omega_{1}$ for the possible three-magnon processes is at the most satisfied at special discrete values of an external control parameter, such as external field magnitude or direction, and will therefore be ignored here. Higher-order terms have larger available phasespace. In forth order, we have the terms

$$
\frac{1}{8} a_{J} \sin [\beta]\left[\left|c_{i}\right|^{4}+c_{i}^{2}\left|c_{i}\right|^{2}\right]
$$

We consider only contributions to $d A_{1} /(d t)$ which in diagrams of the type in Fig. 4 have at least one outgoing mode 1 magnon. Also, we only include scattering events that are compatible with $\omega_{m}>\omega_{1}, \omega_{2}$ for all $m \neq 1,2$. Finally, we exclude scattering events with only one magnon in the thermal bath as 
the requirement on energy conservation at the vertex can in general not be satisfied for such events. In general, these scattering events will then give rise to terms that are more complicated than the ones we have considered previously in that they will not only couple the time evolution of $A_{1}$ and $A_{2}$ with linear terms or terms of the form of the non-linear damping or pumping, and their inclusion in an effective theory would require a much larger parameter set. We will not here further discuss such an effective theory as we are assuming that an expansion to third order is sufficient, and therefore fourthorder terms multiplying $\sin \beta$ can certainly be ignored for small $\beta$. The main reason for discussing these terms is to point out that they alone can cause dependence on angle $\beta$ other than $a_{J} \cos \beta$ and are, for example, responsible for the observed increase[22] in mode-hopping as $\cos \beta$ is decreased.

\subsubsection{Eigenvector phases and mode hopping}

In order for the back-scatter terms to give rise to non-trivial dynamics, i.e., a mode-coupling that will transfer energy between modes 1 and 2, the contributions from Eq. (70) and (73) cannot be all imaginary. In general, the eigenmodes of the linearized equations are all locally elliptical, so that the $y$ and $z$-components $m_{n, y}^{0}$ and $m_{n, z}^{0}$ of the eigenmodes are simply related by

$$
m_{n, z, i}^{0}= \pm \mathrm{i} m_{n, y, i}^{0} f_{n, i}
$$

where $f_{n, i}$ is a real (spatially varying) function. The phase $\varphi_{n, i}$ of the eigenvector $d_{n, i}$ is then

$$
\varphi_{n, i}=\frac{\Im\left[m_{n, y, i}^{0}\right]\left(1 \pm f_{n, i}\right)}{\Re\left[m_{n, y, i}^{0}\right]} .
$$

In some simple systems, such as a single-layer disk (see below), the eigenmodes can be chosen with $m_{n, y, i}^{0}$ real. As a consequence, the eigenvectors $\left|d_{n}\right\rangle$ of such modes are purely real (or imaginary). In such systems the back-scattering terms from Eq. (70) are purely imaginary and do not lead to any non-trivial dynamics. In that case, only the last local term of Eq. (73) can lead to an energy transfer between the modes and concommitant nontrivial dynamics. However, the contributions from Eq. (73) has two small pre-factors, $\alpha_{G} \sim 0.01$, and

$$
\frac{\alpha_{G}}{4}\left(H_{\mathrm{ext}}+H_{d, i, x}^{0}\right)-a_{J} \cos [\beta],
$$

and the latter is small at small driving near the onset of auto-oscillations. For more complicated systems, the eigenmodes acquire a nutation across the area 
of the devices with a non-trival phase relation between different regions of the device for a single eigenmode. As a consequence, $m_{n, y, i}^{0}$ cannot be chosen to be all real (or imaginary), which in turn leads to complex eigenvectors $\left|d_{n}\right\rangle$ and complex back-scattering terms. We will show later, for some specific examples, how the dynamics of an MTJ with two (or three) magnetic layers becomes more complicated than for a single-layer disk as a consequence of the interactions between the magnetic layers and the non-trivial contributions to the back-scattering terms will come from Eq. (70).

Let us consider Eqs. (70) and (73) in some detail as these equation are responsible for the new physics, other than nonlinear damping and pumping, that occurs because of mode interactions. First of all, we note again that $\alpha_{G} \ll|\mathrm{i}|=1$, and also $\left|\alpha_{G}\left(H_{\mathrm{ext}}-N_{x} M_{S}\right) / 4-a_{J} \cos [\beta]\right| \ll 1$ in the regime of small driving near the onset of autooscillations. This means that the prefactors with the imaginary unit i dominate: So long as the eigenvectors are all real or imaginary, Eqs. (70) and (73) will mainly contribute to a frequency shift. However, because of the presence of small but non-zero real prefactors in Eq.(73) this equation will lead to energy transfer between the modes, and the orbits of the system will move away from fixed points and closer to saddle points on the manifold of the dynamics. This then enables mode hopping from orbits in the basin of one fixed points through thermal excitations across saddle points and to orbits in a basin near the other fixed point. These processes will always contribute to mode hopping even if the eigenvectors are all real or imaginary, and the ubiquitous presence of mode hopping is consistent with the observations in Ref. [22], where some mode hopping was always observed. Second, we note that if the eigenvectors are complex, Eq. (70) will lead to mode-coupling that will enable thermally assisted mode hopping through the imaginary prefactors. We will show below, using numerical modeling for an elliptical and circular system, that the presence of the fixed layer in general leads to complex eigenvectors for the modes in the free layer. If the in-plane external field is such that the fixed layer and the free layer magnetizations are parallel or anti-parallel, the eigenvectors are mostly real or imaginary, meaning the phase of the eigenmodes is constant across the free layer. However, as the direction of the external field changes so that the angle between the free layer and fixed layer magnetizations moves away from 0 or $\pi$, the eigenmodes incur some nutation across the free layer with a spatially varying phase difference. This, then, according to Eq. (70), in general leads to increased back-scattering and mode hopping, consistent with observations[22]. 
3.2.6. Numerical examples for circles and ellipses with in-plane external field

We will now illustrate with some specific numerical examples how eigenvectors with non-trivial phases can arise for some simple circular and elliptical systems in the presence of in-plane external fields. We consider two different devices, the first one is a circular devices of diameter $240 \mathrm{~nm}$, and the second one an elliptical device with major axes $240 \mathrm{~nm}$ and $160 \mathrm{~nm}$. The FL in both cases is a $5 \mathrm{~nm}$ thick CoFe layer with a magnetization density of $1200 \mathrm{emu} / \mathrm{cm}^{3}$ and negligible anisotropy energy. We consider three cases for each device: (i) a $5 \mathrm{~nm}$ thick fixed layer exchange biased to an AFM along the $-\hat{x}$-direction with strength $0.8 \mathrm{erg} / \mathrm{cm}^{2}$ and saturation magnetization density $M_{S}=1200 \mathrm{emu} / \mathrm{cm}^{3}$, (ii) fixed layer $M_{S}=200 \mathrm{emu} / \mathrm{cm}^{3}$, and (iii) no fixed layer at all. Case (ii) with low fixed layer magnetization is a simple approximation of the synthetic antiferromagnets that are commonly used in MTJs with small net strayfields affecting the free layer; keeping the exchange bias relatively high at $0.8 \mathrm{erg} / \mathrm{cm}^{2}$ will make the effective exchange bias field very high, but that is not important for the present purposes where we are only interested in modes in the free layer. The exchange coupling in both layers is taken to be $A=1.3 \mu \mathrm{erg} / \mathrm{cm}$. An external magnetic field is applied in the plane of the free layer and can rotate from $\varphi=0^{\circ}$ to $\varphi=90^{\circ}$ relative to the $\hat{x}$-axis. We let the magnetization in the structures relax by integrating the LLG equations using a dimensionless damping of $\alpha=0.2$ on a Cartesian mesh of size $5 \mathrm{~nm} \times 5 \mathrm{~nm} \times 5 \mathrm{~nm}$, calculating magnetostatic interactions by fast Fourier transforms. We then numerically calculate the first 100 eigenmodes by diagonalizing the linearized equations of motion with zero damping. We also performed some test for a finer mesh of $2.5 \mathrm{~nm} \times 2.5 \mathrm{~nm}$ in the plane to ensure that the coarser mesh gave qualitatively and quantitatively correct results.

Figure 5 shows the two lowest modes for the circular and elliptical devices with no fixed layer. As mentioned earlier, one mode is an edge mode (there are actually two edge modes that are nearly degenerate, one with the magnetization at the edges $180^{\circ}$ out of phase, and one with the magnetization in-phase), and one is a Kittel-like mode; for all modes the motion is elliptical with a phase difference of $\pm \pi / 2$ between the $\hat{y}^{\prime}$ and $\hat{z}^{\prime}$ components of the motion, and the phases of the eigenvectors are constant across the free layer. Consequently, the eigenvectors $\left|d_{1}\right\rangle$ and $\left|d_{2}\right\rangle$ are real.

Adding the low-magnetization fixed layer does not change the lowest eigenmodes appreciably. Figure 6 depicts the normalized modulus of the two 


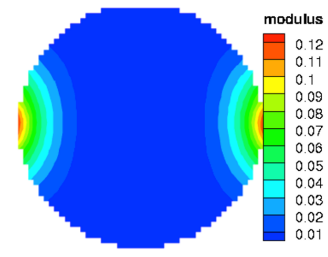

(a)

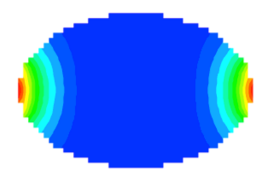

(c)

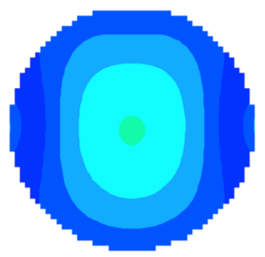

(b)

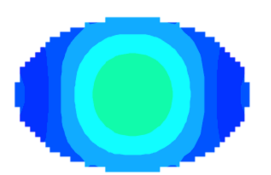

(d)

Figure 5: Normalized magnitude of the two lowest eigenmodes for the circular system with no fixed layer [panels (a) and (b)] and the corresponding elliptical one [panels (c) and (d)]. The external field is along the horizontal axis.

lowest eigenmodes for the circular and elliptical systems. The two lowest eigenmodes are still (near-degenerate) edge modes, the amplitudes of which are shown in panels (a) and (c) of Fig. 6, and the third lowest mode is the Kittel-like mode as shown in panels (b) and (d) in Fig. 6. There is however a difference that emerges in the phases of the eigenmodes, compared to the example with no fixed layer. Figure 7 depicts phase maps for three modes with the field along the horizontal axis [panels (a), (b) and (c)]. Again, the phase difference is constant at $\pm \pi / 2$ across the free layer for the lowest two modes (the arctangent function gives rise to the branchcuts seen in the figure) as shown in the panels (a) and (b) of Fig. 7. But now small, but discernible, nutations of the eigenmodes start to appear, visible as a phase variation of eigenmodes across the surface of the device. Panel (c) of Fig. 7 shows the phase variation of the 18th eigenmode with the field along the horizontal. A small, but clear non-constant phase starts to appear. As the field is rotated relative to the horizontal, the phase variation becomes stronger. Panel (d) of Fig. 7 shows the phase map of sixth eigenmode with the field in the vertical direction. The nutation becomes more pronounced for the elliptical device. Figure 8 shows the phase maps for the second and third (Kittel-like) eigenmodes in panels (a) and (b), and the panels (c) and (d) show, respectively, phase maps for the 12th eigenmode with the field along the horizontal and 


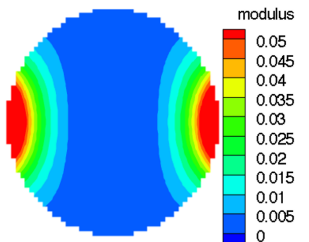

(a)

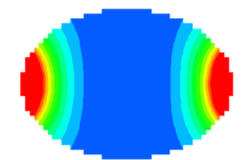

(c)

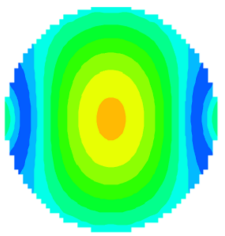

(b)

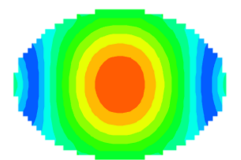

(d)

Figure 6: Normalized magnitude of the two lowest eigenmodes for the circular system with low- $M_{S}$ fixed layer [panels (a) and (b)] and the corresponding elliptical one [panels (c) and (d)]. The external field is along the horizontal axis.

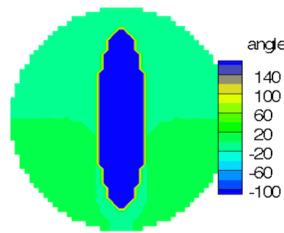

(a)

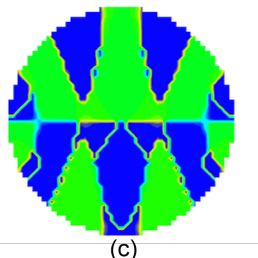

(c)

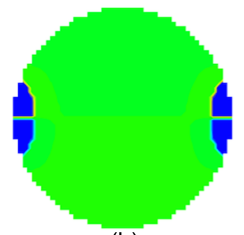

(b)

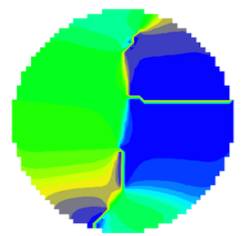

(d)

Figure 7: Panels (a) and (b): Phase map in degrees for the lowest two modes depicted in panels (a) and (b) of Fig. 6. Panel (c): phase of the 18th eigenmode with the field along the horizontal direction. Panel (d): phase of the 7th mode with the field at an angle of $90^{\circ}$. 


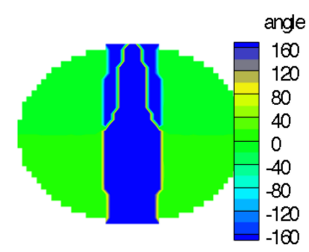

(a)

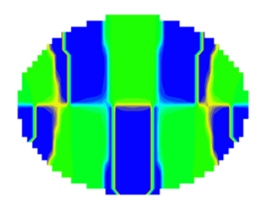

(c)

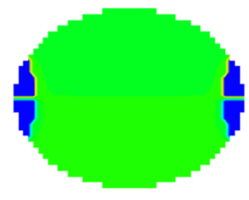

(b)

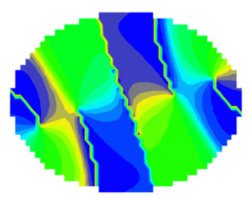

(d)

Figure 8: Panels (a) and (b): phase map in degrees for the lowest two modes depicted in panels (c) and (d) of Fig. 6. Panel (c): phase of the 12th eigenmode with the field along the horizontal direction. Panel $(\mathrm{d})$ : phase of the 9 th mode with the field at an angle of $45^{\circ}$.

the 9 th eigenmode with the field at an angle of $45^{\circ}$.

On the other hand, Fig. 9 show the phase maps for the lowest mode for devices with the fixed layer $M_{S}=1200 \mathrm{emu} / \mathrm{cm}^{3}$ and the field along the horizontal [panels (a) and (c) ] and the field at $45^{\circ}$ to the horizontal, rotating the free layer magnetization approximately $45^{\circ}$ away from the reference layer [panels (b) and (d)]. It is clear from this figure that this mode exhibits considerable nutation with concommitant complex eigenvectors $\left|d_{1}\right\rangle$.

Finally, whether or not modes can cause mode hopping through Eqs. (70) and (73), also depends on the existence of modes with complex eigenvectors that can satisfy the energy conservation expressed by the $\delta$-function in those equations. Because magnon energies are small compared to room temperature thermal energies, basically all magnon modes are populated thermally at room temperature (except for the driven modes with non-equilibrium populations). As pointed out earlier, this provides for an abundance of populated of modes $n$ and modes $m$ such that energy conservation can be satisfied. The finite linewidths of the modes also smear the delta functions which losens the restriction on energy conservation.

We now illustrate the correlation between mode coupling and non-trivial complex eigenmodes using these circular and elliptical model systems. We 

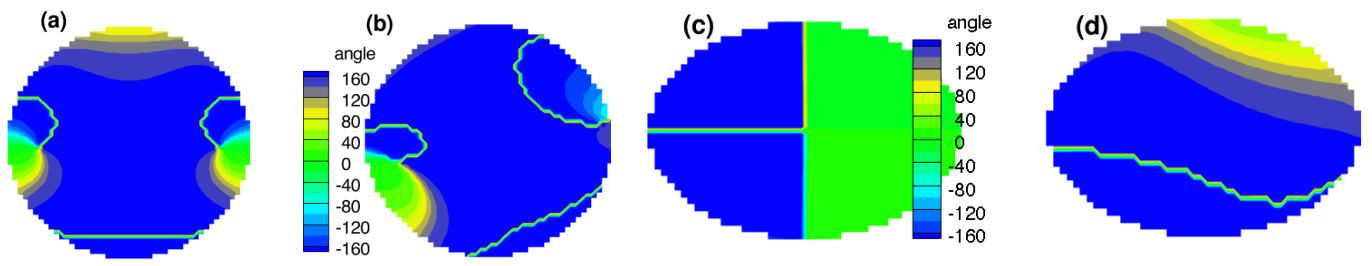

Figure 9: Phase map in degrees for the the Kittel mode with (a) the field along the horizontal axis, and (b) the field at $45^{\circ}$ to the horizontal axis for the circular device. Panels (c) and (d) show the lowest mode for the field along the horizontal axis and at $45^{\circ}$ to the horizontal axis for the elliptical device. For both devices, the fixed layer magnetization density is $M_{S}=1200 \mathrm{emu} / \mathrm{cm}^{3}$.

focus on the mode-coupling that arises from the third-order conservative terms, Eq. (70) and furthermore use a simple local approximation for the demagnetizing tensor, $D_{i, i^{\prime} ; y, y}=-M_{S} N_{y} \delta_{i, i^{\prime}}$ and $D_{i, i^{\prime} ; z, z}=-4 \pi M_{S} \delta_{i, i^{\prime}}$. The mode-coupling term then simplifies to

$$
\frac{d A_{1}}{d t}=\frac{3 \mathrm{i}}{4} A_{2} M_{S}\left[N_{y}+4 \pi\right] \sum_{i, m, n}\left[d_{i, 1}^{*} d_{i, m}^{*} d_{i, 2} d_{i, n}\right] \delta(1+m-2-n) .
$$

We have calculated all the sums of eigenvector terms in Eq. (78) for the model systems, including the 100 lowest eigenmodes for each field angle, and enforcing the energy-conserving delta function by demanding that frequency has to be conserved to within $0.2 \mathrm{GHz}$. Note that the eigenfrequencies for these modes range from a few $\mathrm{GHz}$ to several tens of $\mathrm{GHz}$, so this relaxed frequency conservation corresponds to a small but reasonably realistic linewidth broadening. For the devices without fixed layer, the contributions from these sums are all real, leading to mode hopping only through the small terms in damping or pumping. The results for the devices with nonzero fixed layer magnetization are shown in Fig. 10. There are a couple of clear trends that can be seen in these figures. First, the magnitudes of the sums increase as the fixed layer magnetization density increases, and also increase as the field angle increases (as shown in Fig. 10(b)). Second, as shown in Fig. 10(a), the phase angles of the sums are close to $0^{\circ}$ when the field angle close to zero, and then change as the field angle increases, espcially for the ellipse with $M_{S}=1200 \mathrm{emu} / \mathrm{cm}^{3}$. For a phase angle near $\pm 90^{\circ}$, the mode coupling does not lead to any transfer of energy between the modes, but only contributes 


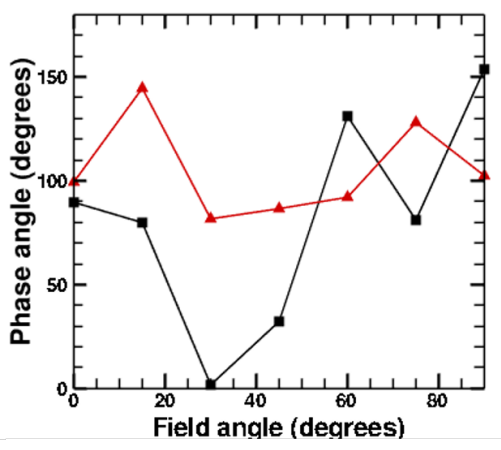

(a)

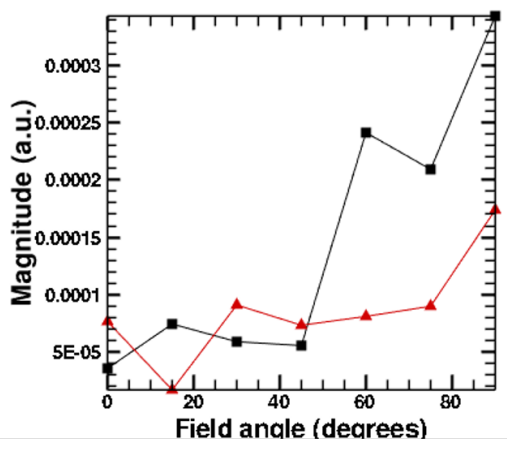

(b)

Figure 10: Phase [panel (a)] and magnitude [panel (b)] of the expression Eq. (78), without the prefactor $\frac{3}{4}\left[N_{y}+4 \pi M_{S}\right]$. Black squares are phase and magnitude for the ellipse with $M_{S}=1200 \mathrm{emu} / \mathrm{cm}^{3}$, and red triangles are for the ellipse with fixed layer $M_{S}=200 \mathrm{emu} / \mathrm{cm}^{3}$.

to a non-linear frequency shift, and the dynamics of the system is near one of the fixed points. As the field angle moves from $90^{\circ}$, the real part of the backscatter term increases with the consequence that the orbits of the system move away from the fixed points and closer to saddle points. This then enables mode hopping through thermal excitations. These observations are consistent with the experimental observations[22].

\section{General equation for mode coupling}

By utilizing the procedure outlined in Sec. IIB, including elimination of the three-magnon terms in a Hamiltonian formulation, the mode equations for modes 1 and 2 up to and including third-order terms then have the most 
general form

$$
\begin{aligned}
\frac{d A_{1}(t)}{d t}= & -\mathrm{i}\left[\eta_{1,1}\left|A_{1}\right|^{2}+\eta_{1,2}\left|A_{2}\right|^{2}\right] A_{1} \\
& -\Gamma_{G, 1}\left[1+P_{1,1}\left|A_{1}\right|^{2}+P_{1,2}\left|A_{2}\right|^{2}\right] A_{1} \\
& +\sigma_{0} I \cos \beta\left[1-Q_{1,1}\left|A_{1}\right|^{2}-Q_{1,2}\left|A_{2}\right|^{2}\right] A_{1}+R_{1,2}(T) A_{2} \\
\frac{d A_{2}(t)}{d t}= & -\mathrm{i}\left[\eta_{2,1}\left|A_{1}\right|^{2}+\eta_{2,2}\left|A_{2}\right|^{2}\right] A_{2} \\
& -\Gamma_{G, 2}\left[1+P_{2,1}\left|A_{1}\right|^{2}+P_{2,2}\left|A_{2}\right|^{2}\right] A_{2} \\
& +\sigma_{0} I \cos \beta\left[1-Q_{1,2}\left|A_{1}\right|^{2}-Q_{2,2}\left|A_{2}\right|^{2}\right] A_{2}+R_{2,1}(T) A_{1}
\end{aligned}
$$

We have noted the temperature dependence of the linear term that arises from scattering of thermally populated modes. In contrast with the nonlinear frequency shift, the non-linear damping and pumping is driven both by local [e.g., lines 5 and 8 in Eq. (72)] as well as non-local terms; of course, the nonlinear damping originates in the magnetostatic interactions, just like the nonlinear frequency shift. Note that Eq. (72) implies that $P_{1,2} \neq P_{2,1}$, that is, the nonlinear damping of mode 1 because of population of mode 2 is in general not the same as the nonlinear damping imparted by a population of mode 1 on mode 2, contrary to the assumption in Ref. [[22]], while the off-diagonal non-linear pumping is symmetric, $Q_{1,2}=Q_{2,1}$.

The thermal populations of modes $n$ and $m$ contributing to back-scattering terms arising from cubic terms have as a consequence that the backscattering terms will have a direct temperature dependence. As the backscattering terms are responsible for bifurcation and mode crossing[27, 26], this implies that the manifold of periodic orbits and fixed points, both stable and unstable ones at saddle points, will shift as the temperature is varied[28]. This is in contrast to the temperature effects that occur when the system is coupled to a thermal bath that gives rise to a stochastic field. Thermal fluctuations will primarily induce mode-hopping over saddle points and thermal excursions around periodic orbits and stable fixed points, but have a small effect on the manifold itself.

Equations (79) and (80) are the equations given by Muduli, Heinonen, and Akerman[22], with the differences that $(i)$ we have written Eqs. (79-80) with our definitions of $\eta_{m, n}, P_{m, n}$, and $Q_{m, n}$ from Sec. III that highlight their symmetry properties and show that the expansion is correctly[12, 15] one in $\left|A_{m}\right|^{2}$ and not in oscillator energy $\omega_{m}\left|A_{m}\right|^{2}$, obviating the factors $\omega_{m}$ used by Muduli, Heinonen, and Akerman[22], and ( $i i)$ that the factors $\Gamma_{G, i}, i=1,2$ 
are (slightly) different for the different modes by a factor of $\omega_{2} / \omega_{1}$, as noted in Sec. 3.2.1.

\section{Summary and Conclusions}

We have here presented detailed derivations of the equations of motions for coupled modes in STOs. In particular, we have shown that the equations governing a system with two dominant modes can be reduced to a set of coupled equations first given by Muduli, Heinonen, and Åkerman[22], and are a generalization of the equations governing a single-mode STO, as given by Slavin and Tiberkevich[12, 15]. We have given explicit expressions for the linear terms and for the cubic terms responsible for nonlinear damping and pumping, as well as for the nonlinear frequency shift, for the examples considered here. We have also confirmed that the off-diagonal non-linear frequency shift and the off-diagonal non-linear damping are symmetric, $\eta_{1,2}=\eta_{2,1}$ and $Q_{1,2}=Q_{2,1}$, while the off-diagonal nonlinear damping is not symmetric, $P_{1,2} \neq P_{2,1}$ for these examples. The linear "back-scattering" term arise from scattering that is possible when there is a bath of modes available, and we have given explicit examples of such terms for our examples. In practice, these terms are difficult to calulate for general geometries and the corresponding coefficients, $R_{1,2}$, and $R_{2,1}$, can probably be treated as parameters in modeling $[25,28]$. We have also concluded that these back-scattering terms have a direct temperature dependence as they involve thermal populations of modes. This implies that manifold of orbits and fixed point will shift as a function of temperature, in addition to temperature effects, such as mode hopping, that may be the consequence of a stochastic field that arises from coupling to a thermal bath. The equations for the coupled modes include additional terms beyond third order that arise when the free layer equilibrium magnetization is not aligned with the fixed layer magnetization. These terms generate additional coupling between modes that provide a physical mechanism for observed increased mode-hopping as the external field is moved away from the direction of the fixed layer magnetization. The intrinsic nonlinear and non-local interactions in the LLG equation that govern the magnetization dynamics give rise to couplings between modes that in turn can generate a wealth of interesting complicated phenomena, such as mode-hopping or mode coexistence. Examples of these phenomena within the framework of the effective driven equations (79) and (80) applied to a nanocontact STO were given by Iacocca et al.[28] with good qualitative and quantitative agreement 
between experimental and modeling results. This fact, together with other observations consistent with our theory $[8,29]$, shows that the theory derived here and the effective equations describing the driven system are the right framework within which to analyze the behavior of STOs both form a fundamental point of view, but also to elucidate their behavior in technological applications.

Work by O.H. was supported by the U.S. Department of Energy (DOE), Office of Science, Division of Materials Sciences and Engineering, and O.H. gratefully acknowledges the computing resources provided on Blues and Fusion, high-performance computing clusters operated by the Laboratory Computing Resource Center at Argonne National Laboratory. S. S.-L. Zhang was supported by NSF Grants DMR-1406568 and partly by Department of Energy, Office of Science, Materials Sciences and Engineering Division through Materials Theory Institute. Yan Zhou acknowledges the support by the Seed Funding Program for Basic Research from the University of Hong Kong, and University Grants Committee of Hong Kong (Contract No. AoE/P-04/08). The authors thank E. Iacocca and R.A. Heinonen for helpful comments on the manuscript.

\section{Appendix A. APPENDIX A}

In general, the magnetization may deviate slightly from being uniform, as is evident in Fig. 2. This can be accounted for in the general formulation. We introduce local coordinates $\left(x_{i}^{\prime}, y_{i}^{\prime}, z_{i}^{\prime}\right)$ such that the local magnetization at site $i^{\prime}$ is along the $x_{i}^{\prime}$ direction. Note that we can without loss of generality assume that $z_{i}^{\prime}=z$. Let $\mathbf{R}_{i}$ be the $3 \times 3$ rotation matrix that transforms the global coordinates $(x, y, z)$ to the local coordinates $\left(x_{i}^{\prime}, y_{i}^{\prime}, z_{i}^{\prime}\right)$ :

$$
\mathbf{R}_{i}=\left(\begin{array}{ccc}
\cos \phi_{i} & -\sin \phi_{i} & 0 \\
\sin \phi_{i} & \cos \phi_{i} & 0 \\
0 & 0 & 1
\end{array}\right)
$$

In terms of the local coordinate, the external field will be rotated by the angle $\phi_{i}$. This willlead to quadratic terms in the Hamiltonian (with small pre-factors $\propto \sin \phi_{i}$ ). The terms involving the spin transfer torque change trivially by $\beta \rightarrow \beta+\phi_{i}$. For the demagnetizing field that contain the interactions between spins at $i$ and $i^{\prime}$, we have in the global coordinates

$$
H_{d, i, \delta}=\sum_{i^{\prime}, \epsilon} D_{i, i^{\prime} ; \delta, \epsilon} \hat{m}_{i^{\prime}, \epsilon} .
$$


We multiply this equation on the left by $\mathbf{R}_{i}$ and insert a resolution of identity $\mathbf{R}_{i^{\prime}}^{-1} \mathbf{R}_{i^{\prime}}$ between $D_{i, i^{\prime} ; \delta, \epsilon}$ and $\hat{m}_{i^{\prime}, \epsilon}$. By re-defining the demagnetizing tensor as

$$
\tilde{D}_{i, i^{\prime} ; \delta, \epsilon}=\sum_{\delta^{\prime}, \epsilon^{\prime}} R_{i ; \delta, \delta^{\prime}} D_{i, i^{\prime} ; \delta^{\prime}, \epsilon^{\prime}} R_{i^{\prime} ; \epsilon^{\prime}, \epsilon}^{-1}
$$

we can proceed and define the Holstein-Primakoff transformation in terms of the magnetization directors in the local coordinates $\left(x_{i}^{\prime}, y_{i}^{\prime}, z_{i}^{\prime}\right)$. On the left hand side, we then have the demagnetizing field in the local coordinates $\left(x_{i}^{\prime}, y_{i}^{\prime}, z_{i}^{\prime}\right)$.

\section{Appendix B. APPENDIX B}

In this appendix, we present the general expressions for the coefficients of the four-magnon terms in Eq. (55). The coefficients of the four-magnon terms derived from the nonresonant three-magnon terms can be written as

$$
\begin{aligned}
W_{i_{1}, i_{2}, i_{3}, i_{4}}= & 2 \sum_{i_{5}}\left(\frac{X_{i_{5}, i_{3}, i_{4}}^{*} X_{i_{5}, i_{1}, i_{2}}}{\omega_{i_{5}}+\omega_{i_{1}}+\omega_{i_{2}}}+\frac{Y_{i_{1}, i_{2}, i_{5}} Y_{i_{5}, i_{3}, i_{4}}+Y_{i_{3}, i_{4}, i_{5}}^{*} Y_{i_{5}, i_{1}, i_{2}}^{*}}{\omega_{i_{5}}-\omega_{i_{1}}-\omega_{i_{2}}}\right. \\
& \left.+\frac{Y_{i_{1}, i_{5}, i_{3}} Y_{i_{5}, i_{2}, i_{4}}+Y_{i_{3}, i_{5}, i_{1}}^{*} Y_{i_{5}, i_{4}, i_{2}}^{*}}{\omega_{i_{5}}+\omega_{i_{2}}-\omega_{i_{4}}}\right)
\end{aligned}
$$

where the coefficients $X$ and $Y$ are given by Eqs. (49) and (50).

The general expression of the coefficients of the resonant four-magnon terms is given by

$$
\begin{aligned}
\Gamma_{i_{1}, i_{2}, i_{3}, i_{4}}=\sum_{i, i^{\prime}} & {\left[F _ { i , i ^ { \prime } } \left(U_{i, i_{1}} U_{i^{\prime}, i_{2}} U_{i, i_{3}}^{*} U_{i^{\prime}, i_{4}}^{*}+V_{i^{\prime}, i_{1}}^{*} V_{i, i_{2}}^{*} V_{i^{\prime}, i_{3}} V_{i, i_{4}}\right.\right.} \\
& \left.+2 U_{i, i_{1}} V_{i, i_{2}}^{*} V_{i^{\prime}, i_{3}} U_{i^{\prime}, i_{4}}^{*}+2 U_{i, i_{1}} V_{i^{\prime}, i_{2}}^{*} V_{i^{\prime}, i_{3}} U_{i, i_{4}}^{*}\right) \\
& +G_{i, i^{\prime}}\left(U_{i, i_{1}} U_{i^{\prime}, i_{2}} U_{i^{\prime}, i_{3}}^{*} U_{i^{\prime}, i_{4}}^{*}+V_{i^{\prime}, i_{1}}^{*} V_{i^{\prime}, i_{2}}^{*} V_{i^{\prime}, i_{3}} V_{i, i_{4}}\right. \\
& +U_{i^{\prime}, i_{1}} U_{i^{\prime}, i_{2}} U_{i, i_{3}}^{*} U_{i^{\prime}, i_{4}}^{*}+V_{i^{\prime}, i_{1}}^{*} V_{i, i_{2}}^{*} V_{i^{\prime}, i_{3}} V_{i^{\prime}, i_{4}} \\
& +2 U_{i, i_{1}} V_{i^{\prime}, i_{2}}^{*} V_{i^{\prime}, i_{3}} U_{i^{\prime}, i_{4}}^{*}+2 U_{i^{\prime}, i_{1}} V_{i^{\prime}, i_{2}}^{*} V_{i, i_{3}} U_{i^{\prime}, i_{4}}^{*} \\
& \left.+2 V_{i^{\prime}, i_{1}}^{*} U_{i^{\prime}, i_{2}} U_{i, i_{3}}^{*} V_{i^{\prime}, i_{4}}+2 V_{i, i_{1}}^{*} U_{i^{\prime}, i_{2}} U_{i^{\prime}, i_{3}}^{*} V_{i^{\prime}, i_{4}}\right) \\
& +B_{i, i^{\prime}}\left(2 U_{i, i_{1}} U_{i^{\prime}, i_{2}} V_{i^{\prime}, i_{3}} U_{i^{\prime}, i_{4}}^{*}+U_{i^{\prime}, i_{1}} U_{i^{\prime}, i_{2}} V_{i, i_{3}} U_{i^{\prime}, i_{4}}^{*}\right. \\
& \left.+U_{i, i_{1}} V_{i^{\prime}, i_{2}}^{*} V_{i^{\prime}, i_{3}} V_{i^{\prime}, i_{4}}+V_{i^{\prime}, i_{1}}^{*} U_{i^{\prime}, i_{2}} V_{i^{\prime}, i_{3}} V_{i, i_{4}}+U_{i^{\prime}, i_{1}} V_{i^{\prime}, i_{2}}^{*} V_{i, i_{3}} V_{i^{\prime}, i_{4}}\right) \\
& +B_{i, i^{\prime}}^{*}\left(2 V_{i^{\prime}, i_{1}}^{*} U_{i^{\prime}, i_{2}} U_{i, i_{3}}^{*} U_{i^{\prime}, i_{4}}^{*}+V_{i, i_{1}}^{*} U_{i^{\prime}, i_{2}} U_{i^{\prime}, i_{3}}^{*} U_{i^{\prime}, i_{4}}^{*}\right. \\
& \left.\left.+V_{i^{\prime}, i_{1}}^{*} V_{i^{\prime}, i_{2}}^{*} U_{i, i_{3}}^{*} V_{i^{\prime}, i_{4}}+V_{i^{\prime}, i_{1}}^{*} V_{i, i_{2}}^{*} V_{i^{\prime}, i_{3}} U_{i^{\prime}, i_{4}}^{*}+V_{i, i_{1}}^{*} V_{i^{\prime}, i_{2}}^{*} U_{i^{\prime}, i_{3}}^{*} V_{i^{\prime}, i_{4}}\right)\right]
\end{aligned}
$$


where $U_{i, i^{\prime}}$ and $V_{i, i^{\prime}}$ are the coefficients of the linear Bogoliubov transformation defined by Eq. (38), and the coefficients $B_{i, i^{\prime}}, F_{i, i^{\prime}}$ and $G_{i, i^{\prime}}$ are given by Eqs. (28), (30) and (31) respectively.

[1] J. C. Slonczewski, Current-driven excitation of magnetic multilayers, J. Magn. Magn. Mater. 159 (1996) L1-L7. doi:10.1016/03048853(96)00062-5.

[2] L. Berger, Emission of spin waves by a magnetic multilayer traversed by a current, prb 54 (1996) 9353-9358. doi:10.1103/PhysRevB.54.9353.

[3] D. C. Ralph, M. D. Stiles, Spin transfer torques, J. Magn. Magn. Mater. 320 (2008) 1190-1216. doi:10.1016/j.jmmm.2007.12.019.

[4] A. V. Nazarov, K. Nikolaev, Z. Gao, H. Cho, D. Song, Microwave generation in $\mathrm{MgO}$ magnetic tunnel junctions due to spin transfer effects (invited), J. Appl. Phys. 103 (7) (2008) 07A503. doi:10.1063/1.2836973.

[5] A. M. Deac, A. Fukushima, H. Kubota, H. Maehara, Y. Suzuki, S. Yuasa, Y. Nagamine, K. Tsunekawa, D. D. Djayaprawira, N. Watanabe, Bias-driven high-power microwave emission from MgO-based tunnel magnetoresistance devices, Nat. Phys. 4 (2008) 803-809. doi:10.1038/nphys1036.

[6] D. Houssameddine, U. Ebels, B. Dieny, K. Garello, J.-P. Michel, B. Delaet, B. Viala, M.-C. Cyrille, J. A. Katine, D. Mauri, Temporal Coherence of $\mathrm{MgO}$ Based Magnetic Tunnel Junction Spin Torque Oscillators, Phys. Rev. Lett. 102 (25) (2009) 257202. doi:10.1103/PhysRevLett.102.257202.

[7] Z. Zeng, K. H. Cheung, H. W. Jiang, I. N. Krivorotov, J. A. Katine, V. Tiberkevich, A. Slavin, Evolution of spin-wave modes in magnetic tunnel junction nanopillars, Phys. Rev. B 82 (10) (2010) 100410. doi:10.1103/PhysRevB.82.100410.

[8] P. Muduli, O. Heinonen, J. Åkerman, Phys. Rev. B 86 (2012) 174408.

[9] F. B. Mancoff, N. D. Rizzo, B. N. Engel, S. Tehrani, Area dependence of high-frequency spin-transfer resonance in giant magnetoresistance contacts up to $300 \mathrm{~nm}$ diameter, Appl. Phys. Lett. 88 (11) (2006) 112507. doi:10.1063/1.2185620. 
[10] W. Rippard, M. Pufall, S. Kaka, S. Russek, T. Silva, Direct-Current Induced Dynamics in Co90Fe10/Ni80Fe20 Point Contacts, Phys. Rev. Lett. 92 (2) (2004) 027201. doi:10.1103/PhysRevLett.92.027201.

[11] T. J. Silva, W. H. Rippard, Developments in nano-oscillators based upon spin-transfer point-contact devices, J. Magn. Magn. Mater. 320 (2008) 1260-1271. doi:10.1016/j.jmmm.2007.12.022.

[12] A. Slavin, V. Tiberkevich, Excitation of spin waves by spin-polarized current in magnetic nano-structures, IEEE Trans. Magn. 44 (2008) 1916-1927. doi:10.1109/TMAG.2008.924537.

[13] V. S. Tiberkevich, A. N. Slavin, J.-V. Kim, Temperature dependence of nonlinear auto-oscillator linewidths: Application to spin-torque nano-oscillators, Phys. Rev. B 78 (9) (2008) 092401. doi:10.1103/PhysRevB.78.092401.

[14] J.-V. Kim, V. Tiberkevich, A. N. Slavin, Generation Linewidth of an Auto-Oscillator with a Nonlinear Frequency Shift: SpinTorque Nano-Oscillator, Phys. Rev. Lett. 100 (2008) 017207. doi:10.1103/PhysRevLett.100.017207.

[15] A. Slavin, V. Tiberkevich, Nonlinear auto-oscillator theory of microwave generation by spin-polarized current, IEEE Trans. Magn. 45 (4) (2009) 1875-1918. doi:10.1109/TMAG.2008.2009935.

[16] D. V. Berkov, N. L. Gorn, Magnetization oscillations induced by a spin-polarized current in a point-contact geometry: Mode hopping and nonlinear damping effects, Phys. Rev. B 76 (14) (2007) 144414. doi:10.1103/PhysRevB.76.144414.

[17] S. Bonetti, V. Puliafito, G. Consolo, V. S. Tiberkevich, A. N. Slavin, J. Åkerman, Power and linewidth of propagating and localized modes in nanocontact spin-torque oscillators, Phys. Rev. B 85 (17) (2012) 174427. doi:10.1103/PhysRevB.85.174427.

[18] S. Bonetti, V. Tiberkevich, G. Consolo, G. Finocchio, P. Muduli, F. Mancoff, A. Slavin, J. Åkerman, Experimental Evidence of SelfLocalized and Propagating Spin Wave Modes in Obliquely Magnetized Current-Driven Nanocontacts, Phys. Rev. Lett. 105 (21) (2010) 217204. doi:10.1103/PhysRevLett.105.217204. 
[19] I. N. Krivorotov, N. C. Emley, R. A. Buhrman, D. C. Ralph, Time-domain studies of very-large-angle magnetization dynamics excited by spin transfer torques, Phys. Rev. B 77 (5) (2008) 054440. doi:10.1103/PhysRevB.77.054440.

[20] K. J. Lee, A. Deac, O. Redon, J. Nozières, B. Dieny, Nat. Mater. 3 (2004) 877.

[21] J. C. Sankey, I. N. Krivorotov, S. I. Kiselev, P. M. Braganca, N. C. Emley, R. A. Buhrman, D. C. Ralph, Mechanisms limiting the coherence time of spontaneous magnetic oscillations driven by dc spin-polarized currents, Phys. Rev. B 72 (22) (2005) 224427. doi:10.1103/PhysRevB.72.224427.

[22] P. K. Muduli, O. G. Heinonen, J. Åkerman, Decoherence and Mode Hopping in a Magnetic Tunnel Junction Based Spin Torque Oscillator, Phys. Rev. Lett. 108 (20) (2012) 207203. doi:10.1103/PhysRevLett.108.207203.

[23] R. Dumas, E. Iacocca, S. Bonetti, S. Sani, S. Mohseni, A. Eklund, J. Persson, O. Heinonen, J. Åkerman, Phys. Rev. Lett. 110 (2013) 257202 .

[24] F. M. de Aguiar, A. Azevedo, S. M. Rezende, Theory of a twomode spin torque nanooscillator, Phys. Rev. B 75 (13) (2007) 132404. doi:10.1103/PhysRevB.75.132404.

[25] O. Heinonen, P. Muduli, E. Iacocca, J. Åkerman, IEEE Trans. Magn. 49 (2013) 4398.

[26] G. van der Sande, L. Gelens, P. Tassin, J. Scirè, A. aand Danckaert, J. Phys. B: At. Mol. Opt. Phys. 41 (2008) 095402.

[27] S. Beri, L. Gelens, M. Mestre, G. van der Sande, G. Verschaffelt, G. Scirè, G. Mezosi, M. Sorel, J. Danckaert, Phys. Rev. Lett. 101 (2008) 093903.

[28] E. Iacocca, O. Heinonen, P. K. Muduli, J. Åkerman, Generation linewidth of mode-hopping spin torque oscillators, Phys. Rev. B 89 (2014) 054402. doi:10.1103/PhysRevB.89.054402.

URL http://link.aps.org/doi/10.1103/PhysRevB.89.054402 
[29] N. Sharma, P. Dürrenfeld, E. Iacocca, O. Heinonen, J. Åkerman, Appl. Phys. Lett. 105 (2014) 132404.

[30] E. Iacocca, P. Dürrenfeld, O. Heinonen, J. Åkerman, R. K. Dumas, Mode-coupling mechanisms in nanocontact spin-torque oscillators, Phys. Rev. B 91 (2015) 104405. doi:10.1103/PhysRevB.91.104405. URL http://link.aps.org/doi/10.1103/PhysRevB.91.104405

[31] O. Heinonen, Y. Zhou, D. Li, Mode coupling in spin torque oscillators, arXiv:1310.6791.

[32] A. Slavin, V. Tiberkevich, Spin wave mode excited by spin-polarized current in a magnetic nanocontact is a standing self-localized wave bullet, Phys. Rev. Lett. 95 (2005) 237201. doi:10.1103/PhysRevLett.95.237201. URL http://link.aps.org/doi/10.1103/PhysRevLett.95.237201

[33] S. Zhang, P. M. Levy, A. Fert, Mechanisms of Spin-Polarized CurrentDriven Magnetization Switching, Phys. Rev. Lett. 88 (23) (2002) 236601. doi:10.1103/PhysRevLett.88.236601.

[34] P. K. Muduli, O. G. Heinonen, J. Åkerman, Bias dependence of perpendicular spin torque and of free- and fixed-layer eigenmodes in MgO-based nanopillars, Phys. Rev. B 83 (18) (2011) 184410. doi:10.1103/PhysRevB.83.184410.

[35] M. Sorel, J. Laybourn, A. Scirè, S. Balle, G. Giuliani, R. Miglierina, S. Donati, Opt. Lett. 27 (2002) 1992.

[36] M. Sorel, G. Giuliani, A. Scirè, R. Miglierina, J. Laybourn, S. Donati, IEEE J. Quantum Electron. 39 (2003) 1187-1195.

[37] V. P. Krasitskii, Canonical transformation in a theory of weakly nonlinear waves with a nondecay dispersion law, Sov. Phys. JETP 71 (1990) 921.

[38] V. P. Krasitskii, On reduced equations in the hamiltonian theory of weakly nonlinear surface waves, J. Fluid Mech. 272 (1994) 1.

[39] V. S. L'vov, Wave Turbulence Under Parametric Excitations: Applications to Magnets, Springer, Berlin, 1994. 
[40] J. Colpa, Diagonalization of the quadratic boson hamiltonian, Physica A: Statistical Mechanics and its Applications 93 (34) (1978) 327 - 353.

[41] A. N. Slavin, P. Kabos, Approximate theory of microwave generation in a current-driven magnetic nanocontact magnetized in an arbitrary direction, IEEE Trans. Magn. 41 (2005) 1264-1273. doi:10.1109/TMAG.2005.845915.

[42] G. Gerhart, E. Bankowski, G. A. Melkov, V. S. Tiberkevich, A. N. Slavin, Angular dependence of the microwave-generation threshold in a nanoscale spin-torque oscillator, Phys. Rev. B 76 (2) (2007) 024437. doi:10.1103/PhysRevB.76.024437.

[43] Z. Li, S. Zhang, Magnetization dynamics with a spin-transfer torque, Phys. Rev. B 68 (2) (2003) 024404. doi:10.1103/PhysRevB.68.024404.

[44] R. Verba, G. Melkov, V. Tiberkevich, A. Slavin, Collective spin-wave excitations in a two-dimensional array of coupled magnetic nanodots, Phys. Rev. B 85 (2012) 014427. doi:10.1103/PhysRevB.85.014427.

URL http://link.aps.org/doi/10.1103/PhysRevB.85.014427

[45] N. Smith, J. Katine, J. Childress, M. Carey, IEEE Trans. Mag. 41 (2005) 2935. 